\title{
Structure and regulation of the cervicovaginal microbiome in a cohort of Afro-Caribbean women
}

Orville St. E Roachford ( $\Delta$ orville.roachford@mycavehill.uwi.edu )

University of the West Indies at Cave Hill Faculty of Science and Technology https://orcid.org/00000003-1389-0797

\section{Angela T Alleyne}

University of the West Indies at Cave Hill Faculty of Science and Technology

Claire Kuelbs

J Craig Venter Institute

Manolito G Torralba

J Craig Venter Institute

Karen E Nelson

$\mathrm{J}$ Craig Venter Institute

\section{Research}

Keywords: cervicovaginal microbiome, Afro-Caribbean women, immune system, vaginal eubiosis

Posted Date: May 14th, 2020

DOI: https://doi.org/10.21203/rs.3.rs-27657/v1

License: (c) (i) This work is licensed under a Creative Commons Attribution 4.0 International License.

Read Full License 
1 Structure and regulation of the cervicovaginal microbiome in a cohort of

2 Afro-Caribbean women

3

4 Orville St. E. Roachford ${ }^{1 *}$, Angela T. Alleyne ${ }^{1}$, Claire Kuelbs ${ }^{3}$, Manolito G. Torralba ${ }^{2}$, and

5 Karen E. Nelson ${ }^{2,3}$

$6{ }^{1}$ Department of Biological and Chemical Sciences, The University of the West Indies, Cave Hill

7 Campus, Bridgetown, BB 11000, BARBADOS.

$8 \quad{ }^{2} J$. Craig Venter Institute, 9605 Medical Center Drive Suite 150, Rockville, MD 20850, USA.

$9{ }^{3}$ J. Craig Venter Institute, 4120 Capricorn Lane, La Jolla, CA 92037, USA.

10

11 *Corresponding address: Department of Biological and Chemical Sciences, The University of

12 the West Indies, Cave Hill Campus, Bridgetown, BB 11000, BARBADOS.

13 Telephone: +1-246-417-4808, Fax: +1-246-417-4325

14 E-mails: orville.roachford@mycavehill.uwi.edu; oster6413@gmail.com

15 ORCID ID: 0000-0003-1389-0797

16

17 Running title: Regulation of cervicovaginal microbiome

18

19

20 


\section{Abstract}

\section{Background}

23 The cervicovaginal microbiome, with its well documented dynamic community state types (CSTs

$24 \mathrm{I}-\mathrm{V})$ is known to interact with the host immune system in a complex regulatory ecosystem that 25 guards against dysbiosis and invading pathogens. However, the regulatory mechanism of the 26 cervicovaginal microbiome in vaginal eubiosis is not well understood. Therefore, we characterized

27 the cervicovaginal microbiome in a cohort of Afro-Caribbean women to the species-strain 28 taxonomic level using high-throughput targeted sequencing and shotgun metagenomics and 29 conceptualized a hypothetical model that aids in understanding vaginal ecosystem regulation.

\section{Results}

31 Compared to targeted 16S rRNA V4 sequencing, whole genome shotgun (WGS) metagenomics

32 offered greater species resolution of the cervicovaginal microbiome in the Afro-Caribbean women 33 cohort. Community state type IV predominated in the microbiome of these women, with Prevotella 34 (13.91\%), Gardnerella (12.14\%) and Lactobacillus $(9.37 \%)$ being the three most abundant genera.

35 For Prevotella and Lactobacillus the most abundant species-strains were P. timonensis DSM 3622865 (5.0\%) and L. iners DSM 13335 (7.0\%), respectively. The less virulent strain of 37 Gardnerella, G. vaginalis 409-05 (8.0\%) was more abundant than G. vaginalis ATCC 14019 38 (4.0\%). In the resistome, 2,753 antimicrobial resistance (AMR) genes consisting of 28 types 39 (mostly tet and Emr; abundances 51\% and 15\%, respectively) which confer resistance to 40 tetracyclines and the macrolide-lincosamide streptogramin B (MLSb phenotype) group were 41 observed. Functional profiling showed a high abundance of biological processes (bacterial-type 
42 flagellum-dependent cell motility, cell adhesion, response to biotic stimulus and quorum sensing)

43 associated with biofilm activity.

\section{Conclusions}

45 Characterized for the first time, the cervicovaginal microbiome in Afro-Caribbean women is 46 predominantly CST IV with the three most abundant taxa consisting of bacterial strains $P$. 47 timonensis DSM 22865, G. vaginalis 409-05 and L. iners DSM 13335. Its resistome had multiple 48 AMR genes that confer resistance to antibiotics commonly used in the treatment of sexually 49 transmitted infections (STIs) and bacterial vaginosis (BV). In this study, the occurrence of biofilm 50 activity within the cervicovaginal microbiome suggested a possible regulatory role. We present a 51 conceptual immuno-munibiome model that advances a mechanistic approach for the structure and 52 regulation of the vaginal ecosystem in the Afro-Caribbean women cohort.

53

54

55

56

57

58

59

60

61

62 


\section{Background}

64 The external orifice (vaginal introitus) of the female reproductive tract communicates directly with 65 another internal cavity (abdominopelvic / peritoneal cavity). Pathogens entering the lower 66 reproductive tract (vagina) can potentially penetrate the cervical canal, enter the uterus, travel

67 along the fallopian tubes into the abdominopelvic cavity and cause diseases such as vaginitis, 68 cervicitis, chorioamnionitis, preterm birth, pelvic inflammatory disease, infertility, peritonitis, 69 sepsis and even death $[1,2,3]$.

70 Two immune defense strategies within the cervicovaginal tract have evolved along with natural

71 physical barriers (cervical mucus plug and vaginal mucoid film) to guard against pathogens; the 72 innate immune system (cytokines and chemokines secretion; activation of leukocytes and the 73 complement cascade) and the adaptive immune system (antigen presentation / T-cell response) [4, $745,6]$. Presumably, vaginal microbes evolved concomitantly, entering a symbiotic interrelationship 75 initially to survive the host's hostile immune system and later into a unique mutualistic host76 microbe relationship, giving rise to the existent cervicovaginal microbiome.

77 Evolutionarily, the fundamental goal of the cervicovaginal microbiome is survival and persistence; 78 achieved by protecting its host, and itself in the presence of adverse intrinsic (e.g. pregnancy, 79 aging, menstruation, obesity) and extrinsic (hormones, drugs, antibiotics, probiotics, coitus,

80 douching, exogenous pathogens) factors. Two hypotheses have been proposed for the evolution of 81 Lactobacillus-dominant cervicovaginal microbiomes involvement in host protection. The first 82 being the "disease risk hypothesis" in which the cervicovaginal microbiome protects against 83 sexually transmitted infections (STIs) that may negatively impact the fitness of its host (and host's 84 offspring) and the second the "obstetric protection hypothesis" which guards against the high risk 
85 of pregnancy and childbirth complications due to microbial pathogens penetrating the endocervical canal [7]. This follows on the long held premise that Lactobacillus species are the principal protective organisms responsible for preventing dysmicrobism and maintaining vaginal health [8, 9]. With regards to persistence and self-protection from adverse local environmental conditions, cervicovaginal microbes perhaps have accomplished such by virtue of niche differentiation, biofilm formation and acquisition of antimicrobial resistance genes $[10,11,12]$.

Contrary to past dogma, recent research has reported normal vaginal health in some women whose vaginal microbiomes consist of a low abundance (or depletion) of Lactobacillus species, high abundance of obligate anaerobes, $G$. vaginalis or known pathogens $[13,14,15,16,17]$. In addition, overgrowth of vaginal Lactobacillus is known to cause cytolytic vaginosis characterised by vaginal irritation and abnormal discharge [18].

Deep sequencing of the female cervicovaginal tract has shown that the latter harbours an extremely diverse microbiome whose composition is both temporal and individual-specific [19, 20]. However, among women of varying ethnicity and age, and regardless of the number of women under study, this diversity seems to have compositional and structural constraints $[13,16,21,22$, $23,24,25]$. Hence, the recognition of five distinct community state types (CSTs): I, II, III, IV and V, where CST I is predominantly of Lactobacillus crispatus, and CSTs II, III and V dominated by L. gasseri, L. iners and L. jensenii, respectively. CST IV is described as a heterogeneous group dominated by a high abundance of anaerobes such as Prevotella, Dialister, Atopobium, Gardnerella, Megasphaera, Peptoniphilus, Sneathia, Eggerthela, Aerococcus, Finegoldia and Mobiluncus $[17,19]$. Therefore, regulation of the cervicovaginal microbial composition with its multitude of symbiotic interrelationships and host-microbe interactions is complex. Additionally, disease causation by pathobionts and exogenous pathogens remains poorly understood. An in- 
depth assessment of the characteristics (microbial function, composition and structure) of the vaginal ecosystem, its resistome and local immune inflammatory responses are of principal importance in understanding regulation of the cervicovaginal microbiome, vaginal health and disease prevention. The cervicovaginal microbiome has been well characterized using 16S rRNA next-generation sequencing (NGS) technologies for women of the Americas (North, Central and South), Europe, Asia and Africa but not for Caribbean women to date. Consequently, the aim of this study was to fully characterize the cervicovaginal microbiome of Afro-Caribbean women using 16S rRNA V4 and whole genome shotgun metagenomics. In addition, we sought to introduce a conceptual hypothesis for the regulation and structure of the cervicovaginal microbiome.

\section{Materials and Methods}

\section{Participants and sample collection}

Eighteen women (selected via simple random sampling) participated in the study between February and April 2018 following signed informed consent. Their health status was determined in a general clinic. All participants were Afro-Caribbean women between the ages of 18-74, not pregnant and without any physical disabilities, concomitant microbial infections or abnormal vaginal symptoms. For participation in this study, all women were required to refrain from vaginal douching (1 week prior to sampling), avoid antibiotic therapy (6 weeks prior to sampling), not engage in coitus and have completed menses by at least 3 days prior to sampling.

A cervicovaginal lavage procedure was carried out on each woman after taking a brief medical history and giving instructions for the sampling procedure. The lavage procedure involved the passage of a stainless-steel Cusco speculum into the vagina to visualize the cervix and related structures. Twelve millilitres $(12 \mathrm{ml})$ of sterile phosphate buffer solution (PBS) with $\mathrm{pH} 7.4$ was 
131 syringed into the vagina and re-extracted after 1 minute by way of disposable sterile syringes.

132 Approximately $10 \mathrm{ml}$ of the cervicovaginal washings (CVWs) were transferred to $15 \mathrm{ml}$ Falcon

133 tubes, placed on ice and transferred directly for storage at $-80^{\circ} \mathrm{C}$ within 1 hour of collection.

134 Genomic DNA Extraction

135 CVWs were thawed and $1.0 \mathrm{ml}$ aliquots were placed into sterile $1.5 \mathrm{ml}$ Eppendorf microcentrifuge 136 tubes stored on ice. The samples were centrifuged at room temperature for 6 min at 13, $500 \mathrm{rpm}$.

137 Supernatant was removed, and another $1.0 \mathrm{ml}$ of the CVW sample added to the pellet for re138 centrifugation at 13,500 $\mathrm{rpm}$ for $6 \mathrm{~min}$. This procedure was repeated thrice, resulting in the total 139 use of $5.0 \mathrm{ml}$ of CVW. Pellets were stored at $-20^{\circ} \mathrm{C}$ prior to further processing. DNA was extracted 140 from pellets using InstaGene ${ }^{\mathrm{TM}}$ Matrix (Bio-Rad Laboratories, USA). InstaGene ${ }^{\mathrm{TM}}$ Matrix $(200 \mu 1)$

141 was added to the pellets and incubated at $56{ }^{\circ} \mathrm{C}$ for $30 \mathrm{~min}$. The samples were vortexed at high 142 speed for $10 \mathrm{~s}$ and then placed in a boiling water bath for $8 \mathrm{~min}$. Samples were vortexed again for $14310 \mathrm{~s}$ and centrifuged at $12,000 \mathrm{rpm}$ for $5 \mathrm{~min}$. The supernatant was removed and stored at $-20^{\circ} \mathrm{C}$ 144 prior to shipping to J. Craig Venter Institute (JCVI), California, USA for Illumina sequencing. 145 Qualification and quantification of extracted DNA was determined by $2.0 \%$ gel electrophoresis 146 and nanodrop spectrophotometry (NanoDrop® Technologies, Wilmington, DE, USA) at $260 \mathrm{~nm}$.

\section{$147 \quad$ 16S rDNA V4 sequencing by MiSeq Illumina}

148 DNA extracted from the cervicovaginal samples was amplified using custom adaptor ligated 149 primers (primers 515F 5'- GTGCCAGCMGCCGCGGTAA-3' and 806R 5'150 GGACTACHVGGGTWTCTAAT-3') that target the V4 hypervariable region of the 16S rRNA 151 gene [26]. Included in the custom primers, were i5 and i7 adaptor sequences for Illumina MiSeq 152 pyrosequencing. Unique $8 \mathrm{bp}$ indices were also attached to the primers giving each sample its own 
153 unique barcode pair. Incorporation of adaptors and index sequences onto the primers at the PCR

154 stage minimalized the loss of sequence data in comparison to earlier methods that ligated the 155 adaptors to each amplicon post-amplification [27]. All sequence reads generated by this method 156 were of the same 5'-3' orientation. Extracted DNA (approximately 100ng/ $\mu$ l) was used to generate 157 amplicons using Platinum Taq polymerase (Life Technologies, CA, USA) under the following 158 cycling conditions: $95^{\circ} \mathrm{C}$ for $5 \mathrm{~min}$ for an initial denaturing step, $95^{\circ} \mathrm{C}$ for $30 \mathrm{~s}, 57^{\circ} \mathrm{C}$ for $30 \mathrm{~s}, 72^{\circ} \mathrm{C}$ 159 for $30 \mathrm{~s}$ for 35 cycles, followed by a final elongation step at $72^{\circ} \mathrm{C}$ for 7 min and stored at $4{ }^{\circ} \mathrm{C}$. 160 Amplicon purification was performed using the QIAquick PCR purification kit (Qiagen, Valencia, 161 CA, USA), quantified using Tecan fluorometric methodology (Tecan Group, Mannedorf, 162 Switzerland), normalized and then pooled in preparation for paired-end Illumina MiSeq 163 sequencing using V2 chemistry dual index 2x250 bp format (Roche, Branford, CT, USA) as per 164 the manufacturer's protocol.

\section{WGS metagenomic library construction and sequencing}

166 Sample DNA was assessed for high quality and selected for high molecular weight in preparation 167 for library construction using agarose gel electrophoresis. The sample DNA was then sheared 168 using the Covaris ultrasonicator instrument (Covaris, Woburn, Massachusetts, USA). Sheared 169 DNA was quantified, and size selected using NEBNext® Library Quant Kit for Illumina® $($ New 170 England Biolabs, Ipswich, MA, USA) according to manufacturers' specifications. Sample DNA 171 was barcoded to allow for multiplexing of several samples per sequencing run using NexteraXT 172 Index kit (Illumina, San Diego, CA, USA). Metagenomic libraries were sequenced using Illumina 173 NextSeq 500 following standard manufacturer's specifications. Approximately 1-3 Gbp of 174 sequence data per sample was generated to obtain significant functional information for 175 comparative analyses. 
177 Quality control checks on raw Illumina sequence data derived from WGS-metagenomic 178 sequencing was carried out using FastQC v 0.10.1 [28]. All metagenomic sequencing data was 179 processed and analyzed using CLC Microbial Genomics Pro Suite (Qiagen Bioinformatics, 180 Redwood City, CA, USA) with default settings and the contigLCA algorithm within MG-RAST 181 v4.0.3 [29]. Sequences with high Phred scores $(\mathrm{Q}>20)$ were demultiplexed, trimmed of primers 182 and adaptors, as well as filtered of short reads and chimeras. Host sequences were mapped against 183 the reference human genome (GRCh38) and removed. Sequence reads were binned into 184 operational taxonomic units (OTUs) defined at 99\% similarity and matched against 16S rRNA sequences within Greengenes v13_8 99\% database. OTUs were also assigned to taxa at the genus 186 and species level using SeqMatch version 3 in the online Ribosomal Database Project (RDP: 187 release 11_5) [30]. Species diversity within samples and between samples were measured using 188 alpha diversity metrics (Chao 1 bias-corrected, Simpson's index and Shannon entropy) and beta 189 diversity metrics (Bray-Curtis, Jaccard, Euclidean, weighted and unweighted UniFrac), 190 respectively. Beta diversity was visualized with three-dimensional (3D) principal coordinates 191 analysis (PCoA) within CLC Microbial Genomics Module.

\section{Statistical, functional and antibiotic resistance analysis of the WGS metagenomic dataset}

193 Statistical analyses were performed using PERMANOVA (permutational multivariate analysis of 194 variance, also known as a non-parametric MANOVA) [31] in CLC Genomics Workbench v20 195 (https://digitalinsights.qiagen.com). Reads from whole metagenome sequencing dataset were 196 assembled into contigs and annotated using the De Novo Assembled Metagenome and Find

197 Prokaryote Genes tools in CLC Microbial Genomics Module (Qiagen Bioinformatics, Redwood 198 City, CA, USA), respectively. All annotated coding sequences (CDS) with protein families (Pfam) 
199 and Gene Ontology (GO) terms were searched against Pfam v32 and GO databases v1.2 [32, 33,

200 34]. Antimicrobial resistance (AMR) genes within the whole genome shotgun metagenomic

201 dataset of the Afro-Caribbean women were identified using Find Resistance with ShortBRED

202 (FRSB) tool, similar to ShortBRED [35]. DIAMOND v0.9.31 within the FRSB tool (CLC

203 Microbial Genomics Module) was used to match queried sequences against the QMI-AR Peptide

204 Marker database (released 2019-11). All default settings in the FRSB tool were retained except for

205 the parameter "more sensitive search" in order to run DIAMOND at its highest sensitive mode.

206 AMR genes were cross-checked against entries in Comprehensive Antibiotic Resistance Database

207 (CARD) [36].

\section{Results}

$209 \quad$ Read and OTU Statistics

210 Following quality control and removal of human host reads, Illumina NextSeq WGS metagenome

211 sequencing of the bacterial DNA from 18 cervicovaginal samples yielded a total of 181,820,742

212 sequences. Data from 3 samples were unsuitable for metagenomic analysis. The average number

213 of high-quality sequences (Average Phred quality score 34) was 12,121,383 (ranging from

$2144,637,282-26,540,452)$ per sample. The average \% GC content and sequence length were $44 \%$

215 and 151bp, respectively (Supplementary Table S1). For comparison, Illumina MiSeq sequencing

216 of the 16S rRNA V4 hypervariable region was also used to determine the composition of 18

217 cervicovaginal samples of the Afro-Caribbean cohort studied. After filtration of host reads and

218 removal of chimeric sequences, the 18 samples produced a total of 361,723 read pairs in

219 operational taxonomic units (OTUs) with an average read length of 251bp (Supplementary Table 220 S2). 


\section{Taxonomic allocation of OTUs}

222 Removal of short reads from the 361,723 read pairs resulted in an input dataset of 99,322 reads 223 which when filtered yielded 2,141 OTUs. The latter were matched (99\% similarity) against 224 Greengenes v13_8 99\% (16S rRNA) database and clustered into 468 OTUs of which 46 were de 225 novo. Each of the 468 OTUs was assigned to a given taxon, yielding 329 taxa at the genus level. 226 These were further resolved into 236 species based on the highest similarity score (S_ab) as 227 determined by SeqMatch version 3 in the online Ribosomal Database Project (RDP: release 11_5), 228 shown in Supplementary Tables S3 and S4. Of the 236 species, 50\% had matches with similarity 229 scores greater than 0.970 . In addition, $60 \%$ of all species were identified as part of human vagina 230 microbiota while $25 \%, 10 \%$ and $5 \%$ were of gut, oral and non-human (animal, soil, water, sludge 231 etc.) microbial communities, respectively. At the kingdom level, the 15 WGS metagenomes 232 analysed by the online MG-RAST database were on average composed of $86.70 \%$ bacteria, $23313.24 \%$ eukaryotes, $0.04 \%$ viruses and $0.02 \%$ archaea. The contigLCA algorithm within MG234 RAST identified DNA sequences from the sample that shared similarity to sequences at the genus

235 level from humans and Asian monkeys (genus Macaca), ranging from 5.81-66.14\% and 0.94$23612.75 \%$, respectively (https://www.mg-rast.org/linkin.cgi?project=mgp88579). Due to its inability 237 to filter human and Macaca DNA sequences, MG-RAST was not used for further metagenomic 238 analyses. In metagenomic analyses performed with Microbial Genomics Module (CLC genomics 239 workbench 12) human host reads were completely removed and the average composition of the 240 WGS reads (kingdom level) for bacteria and archaea were $99.88 \%$ and $0.12 \%$, respectively. In 241 subsequent analyses, only the bacterial component was used (Supplementary Table S5). 
243 WGS metagenomic analysis was successful for 15 of the 18 cervicovaginal samples compared to 244 16S rRNA V4 sequencing which was successful for all 18 samples. This reflects the ability of the 245 latter sequencing technique to amplify $16 \mathrm{~S}$ rRNA V4 within samples of limited bacterial DNA 246 concentration. In contrast to samples sequenced using 16S rRNA V4, samples sequenced by WGS 247 metagenomics were noted to have a high abundance of Tetrasphaera japonica T1-X7. The next 248 most abundant species were of the genera Lactobacillus, Prevotella, Gardnerella, Anaerococcus, 249 Megasphaera, Shuttleworthia, Sneathia and Porphyromonas. However, the cervicovaginal 250 microbial communities analysed in the absence of $T$. japonica $\mathrm{T} 1-\mathrm{X} 7$ as determined by $16 \mathrm{~S}$ rRNA 251 V4 and WGS were similar (Fig. 1). Taxa identified by 16S rRNA V4 sequences were comparable 252 to that by WGS, with discrepancies occurring mainly at species-level. WGS sequencing offered 253 greater and more accurate resolution at the species-level. Taxonomically, WGS was better able to 254 resolve species to strain-level by identifying 301 species (211 strains) compared to 236 (3 strains) 255 identified by $16 \mathrm{~S}$ rRNA V4 sequencing.

Microbial diversity and composition of the cervicovaginal microbiomes based on16S rRNA

$257 \quad$ V4 sequencing

258 The alpha diversity (which expresses the number of species represented within a given 259 cervicovaginal microbiome) as measured by chao-1 bias-corrected, Shannon entropy and the 260 Simpson's index metrics showed a wide range of heterogeneity (species diversity) within the 18 261 cervicovaginal microbiomes. Generally, there was no distinctive clustering of the cervicovaginal 262 microbiomes based on any of the three metrics used (Fig. 2A and Supplementary Figure S1). 263 Similarly, when microbial diversity between the 18 cervicovaginal microbiomes were compared 264 using five different $\beta$ - diversity metrics (Bray-Curtis, Jaccard, Euclidean, weighted and 265 unweighted UniFrac) and visualized by three-dimensional (3D) Principal Coordinates Analysis 
266 (PCoA), no significant clustering of the cervicovaginal communities was observed (Figs. 2B, 2C 267 and Supplementary Figure S1).

268 Taxa with a relative abundance as low as 5.53e-4 \% of the microbial community were detected by 269 16S rRNA V4 sequencing. The most abundant taxa with coverage greater than $0.35 \%$ are shown 270 in the heatmap in Fig. 3 which also shows the diversity of these taxa in each cervicovaginal 271 microbiome. These taxa are of the genera Lactobacillus, Prevotella, Gardnerella, Anaerococcus, 272 Megasphaera, Sneathia, Dialister, Shuttleworthia, Porphyromonas, Veillonella and Howardella. 273 For the microbiome data, normalised taxon abundances were clustered by Euclidean distance. 274 Prevotella, Lactobacillus and Gardnerella were present in all women. However, Prevotella was 275 the most abundant (mean abundance $23.06 \%$, maximum $71 \%$ when dominant), followed by 276 Lactobacillus (mean abundance $22.26 \%$, maximum 98\% when dominant). For Gardnerella, the 277 mean abundance and maximum abundance when dominant within a microbiome were $10 \%$ and $27863 \%$, respectively. Shuttleworthia was present in $94 \%$ of women and had a mean abundance of $27914 \%$ with a maximum abundance of $83 \%$ when dominant. It should be noted Shuttleworthia shares $28089 \%$ of its $16 \mathrm{~S}$ rRNA gene V4 sequence with that of bacterial vaginosis-associated bacterium 1 281 (BVAB1) [37]. All other genera had mean abundances $\leq 5 \%$ and were present in $11 \%$ to $78 \%$ of 282 all women. Among the 18 women, the most dominant Lactobacillus species was L. iners (19.19\% 283 mean abundance). Less abundant Lactobacillus species present across the cervicovaginal 284 microbiomes were L. crispatus, L. jensenii, L. fornicalis, L. vaginalis, L. rhamnosus, L. 285 coleohominis, L. intestinalis and L. kitasatonis. Also identified within the cervicovaginal 286 microbiomes of the women were rarer (<0.05\% mean abundance) Lactobacillus species such as $L$. 287 reuteri, L. helveticus, L. ultunensis, L. kalixensis and L. zaea (Supplementary Tables S3 and S6). 
Microbial diversity and composition of the cervicovaginal microbiomes based on shotgun whole genome (WGS) sequencing

Bray-Curtis, Jaccard and Euclidean-based Principal Coordinates Analyses (PCoA) did not reveal any distinct clustering of cervicovaginal communities with respect to the health status of the 15 women. Euclidean-based PCoA showed a cluster which consisted of cervicovaginal communities from both cohorts, however, the significance of this is not clear. In $40 \%$ of the cervicovaginal samples analyzed by WGS metagenomics, a large fraction of species remained undiscovered as reflected by the absence of plateaus in Chao-1 corrected, Shannon entropy and Simpson's indexbased alpha diversity measures (Supplementary Figure S1). However, WGS metagenomics identified a much larger number of species per sample compared to 16S rRNA V4 sequencing.

T. japonica T1-X7, a polyphosphate accumulating Gram-positive cocci, was the predominant organism found in the 15 cervicovaginal samples following WGS metagenomic analysis but this is believed to be a contaminant, possibly related to the previous run of the Illumina sequencer on sludge samples. Tetrasphaera are normally isolated from activated sludge in wastewater treatment systems with enhanced biological phosphorus removal (EBPR) [38, 39] though a species, Tetrasphaera remsis, has reportedly been isolated from air (40)]. Not considering $T$. japonica T1X7, and classifying percentage relative abundance in accordance with Ranjan et al. [41], the most abundant taxa (i.e. taxa with abundances $>1.00 \%$ ) were Prevotella spp. (13.91\%), Gardnerella spp. (12.14\%), Lactobacillus spp. (9.37\%), Mobiluncus spp.(7.15\%), Sneathia spp.(5.59\%), Megasphaera genomosp (3.00\%), Atopobium spp.(2.31\%), Bifidobacterium breve (2.00\%), Mageeibacillus indolicus (2.00\%) and Porphyromonas asaccharolytica (2.00\%). Other taxa with low $(0.01-0.49 \%)$, moderate $(0.05-0.49 \%)$ and high $(>0.50-0.99 \%)$ abundances are featured in Table 1 (Supplementary Tables S5 and S6). A greater number of species/strains were identified 
311 for Prevotella (36/34) than for Lactobacillus (22/15) or any other genera. The most common

312 Prevotella species/strains were P. timonensis 4401737, P. amnii DSM 23384, P. bivia DSM 20514

313 and $P$. ihumii with mean abundances of $5.00 \%, 3.00 \%, 2.00 \%$ and $1.00 \%$, respectively. The four

314 most abundant Lactobacillus species/strains identified were L. iners DSM 13335, L. crispatus ST1,

315 L. gasseri and L. jensenii with mean abundances of $7.00 \%, 2.00 \%, 0.14 \%$ and $0.08 \%$, respectively

316 (supplementary Table S5). Of note, was the identification of Mycoplasma species-strains; $M$.

317 hominis ATCC 23144, M. gallisepticum str. R, M. flocculare ATCC 27399 and M. alligatoris

318 A21JP2. M. hominis ATCC 23144, the only human associated Mycoplasma identified, had a mean

319 abundance of $0.12 \%$. Based on the metadata (Supplementary Table S7), 47\% of the participants in

320 the study were noted to have systemic lupus erythematosus (SLE). Hence, we compared the

321 cervicovaginal microbial compositions of women with and without SLE by performing a

322 PERMANOVA analysis [31]. The analysis for the 3 most abundant species/strains (Prevotella,

323 Gardnerella, Lactobacillus) and Mycoplasma is detailed in Supplementary Table S8. Of

324 Prevotella species, only P. brevis ATCC 19188, P.oryzae DSM 17970 and P. paludivivens DSM

32517968 were more significantly abundant in the SLE group with FDR (false discovery rate) p-values

$3260.02,0.02,0.01$ and $\log _{2}$ fold changes of $7.11,7.11,7.41$, respectively. L. crispatus ST1

327 (representing CST I) was significantly more abundant in the cervicovaginal microbiomes of

328 women without SLE having FDR p-value 0.02 and $\log _{2}$ fold change of 6.72. While L. iners DSM

32913335 (CST III) was more abundant in the SLE group with FDR p-value 0.02 and a $\log _{2}$ fold

330 change of 4.09. There were no significant differences in the abundancies of G. vaginalis 409-05,

331 G. vaginalis ATCC 23114 and M. hominis ATCC 23114 (FDR p-values 0.11, 0.82 and 0.17,

332 respectively). Whether there is a meaningful correlation between Prevotella species, L. iners DSM 
13335 and SLE would require a large high-powered study due to the age disparity of the two groups, sampling size and associated confounding factors (such as parity and co-morbidities). Function of the cervicovaginal microbiome

Functional analysis (genome annotation) of the cervicovaginal microbiome whole metagenome shotgun sequencing dataset using Pfam (Protein families) and Gene Ontology (GO) databases generated a set of 98 functional profiles for the 15 cervicovaginal samples with the following ontologies: biological processes (38), cellular components (25) and molecular functions (35). The biological functions which were visualized in the form of a heatmap (Supplementary Figure S2 and Table S9) highlighted the differential gene expressions and associated biological functions, in terms of relative abundance, within and amongst the cervicovaginal microbiomes of the AfroCaribbean women. Of the biological processes, there was upregulation of cell adhesion, bacterialtype flagellum-dependent cell motility, sporulation, quorum sensing, response to biotic stimulus, carbohydrate binding, viral processes and host cell viral entry. Among the more abundant molecular functions were peroxidase and antioxidant activities, electron transfer activity, and amino acid and iron-sulphur cluster binding. Cell well, outer membrane and bacterial-type flagellum were some of the more abundant cellular components.

\section{Antimicrobial resistance genes of the cervicovaginal microbiome}

Among the cervicovaginal shotgun metagenomes of the 15 Afro-Caribbean women, 2,753 antimicrobial resistance (AMR) genes consisting of 28 types (ileS, IsaC, mel, tet32, tetM, tetO, tetQ, tetS, tetW, rpoB, CfxA2, catl, ErmA, ErmB, ErmF, ErmT, ErmX, emrB, emrK, yegN, MexW, patB, TolC, YojI, AcrS, cpxA, gadX and hns) were detected (Supplementary Table S10). This meta-resistome conferred resistance to a number of drug classes (tetracyclines, macrolides, lincosamides, penicillins, phenicols, aminoglycosides, quinolones, rifamycins and mupirocin). 
356 The relative abundances of the 14 most abundant AMR genes are visualized in the area chart (Fig.

357 4). The most abundant drug class was tetracyclines (51\%), followed by the macrolide-lincosamide 358 streptogramin B (MLSb phenotype) group with a relative abundancy of $15 \%$. This group consisted 359 of common antibiotics such as azithromycin, clindamycin and nitroimidazoles. Antibiotic resistance genes tet $M$, tet $Q$ and tet $W$ were noted to be associated with conjugate and non-conjugate transposons. The tet $O$ genes were associated with conjugative plasmids, genetic elements that are 362 involved in horizontal gene transfer (HGT) between microbial species. Also, identified within the 363 meta-resistome were $C f x A 2$, a class A beta-lactamase AMR gene, found in Prevotella intermedia, 364 and antimicrobial resistance genes ileS and rpoB found in the lactic acid producing 365 Bifidobacterium species. Several mechanisms of antibiotic resistance were identified. The most 366 common resistance mechanism found in this study was antibiotic target protection mediated by 367 tetracycline-resistant ribosomal protection proteins (tet32, tetM, tetQ, tetO and tetW). Resistance 368 genes tet32, tetM, tet $O$ and tet $W$ have been found in the Firmicutes (consisting of Gram-positive 369 genera such as Lactobacillus, Streptococcus and Megasphaera. Whereas, the tetQ gene is found 370 in Gram-negative bacteria such as Prevotella and Porphyromonas of the Bacteroidetes phylum $371[42,43]$. Two other AMR mechanisms were protein synthesis inhibition by mutational alterations 372 in the erm genes (ErmA, ErmB, ErmF, ErmT and ErmX ) that encode Erm 23S ribosomal RNA 373 methyltransferase and confer antibiotic resistance to the macrolide-lincosamide streptogramin B 374 group, and the efflux pump mechanism in which antibiotics are transported out of the bacterial 375 cells. For example, TolC an outer membrane efflux protein subunit of multidrug efflux complexes 376 in Gram negative bacteria pumps aminoglycosides, penicillins, cephalosporins, fluoroquinolones 377 and nitroimidazoles out of the cells. Other resistance genes within the cervicovaginal microbiomes 
378 of the Afro-Caribbean women involving antibiotic efflux were emrB, emrK, yegN, MexW, patB 379 and YojI.

380 Discussion

381 This research presents the first characterization and comparative metagenomic analysis of the 382 cervicovaginal microbiomes of a group of Afro-Caribbean women based on high-throughput next 383 generation sequencing (targeted 16S rRNA V4 sequencing and whole genome shotgun 384 metagenomics).

\section{S rRNA V4 sequencing vs whole genome shotgun metagenomics}

386 Based on our results, WGS metagenomic sequencing resulted in a greater depth of sequencing and 387 produced greater phylogenetic resolution at the species/strain levels as seen in other studies [44, 388 45]. This is not surprising as the extent of microbial diversity determined by $16 \mathrm{~S}$ rRNA V4 389 sequencing is limited by the availability of species-strain directed primers and the short sequence 390 area (V4) covered [41, 46, 47]. In both cases, there was some misidentification of species. This is 391 due to the occurrence of base calling errors, point errors in reads, artificial replicates, over392 represented reads, GC skewing as well as inherent errors embedded in bioinformatic algorithms 393 within databases such as Greengenes and RDP that were used for taxonomic classification. 394 However, WGS was the better technique for studying the vaginal microbiome for three reasons. 395 Firstly, it allowed for the detection of rare and unknown microbes that might play a significant 396 role in vaginal dysbiosis and associated diseases. Secondly, it can offer more consistent 397 phylogenetic resolution compared to partial 16S rRNA sequences, as the various hypervariable 398 regions of the $16 \mathrm{~S}$ rRNA gene have different evolutionary rates and can produce varied 399 phylogenetic resolutions [48]. Thirdly, WGS better differentiated species to the strain level. This 
400 is particularly important in understanding the cervicovaginal microbial interrelationships as

401 species-specific strains display different microbial inhibitory effects. For example, L. gasseri strain

402 KS123.1 has no inhibitory effect on G. vaginalis and P. bivia, whereas L. gasseri strain KS120.1

403 does [49].

\section{Cervicovaginal microbiome of Afro-Caribbean women}

405 The composition of the cervicovaginal microbiomes of the group of Afro-Caribbean women was

406 found to be individual-specific regardless of the sequencing technique utilized (16S and WGS).

407 This specificity is in keeping with research findings from other studies involving large numbers of

408 women $[16,24]$. The cervicovaginal microbiomes showed a wide range of microbial richness

409 within samples (Alpha diversity) and the level of diversity was comparable among the women

410 (Beta diversity). Species diversity was more pronounced with WGS metagenomic sequencing.

411 About $25 \%$ of the bacteria identified in the cervicovaginal samples were associated with the human

412 gut. Previous studies have documented microbes that are common to both microbiomes, with the

413 gut being a likely source for vaginal colonization [16, 50]. Using 16S rRNA V4 sequencing, 4 out

414 of the 5 CSTs previously defined by Ravel et al. [17], were recognised: CST's I (L. crispatus), III

415 (L. iners), IV (heterogenous microbes) and V (L. jensenii). Whereas for WGS metagenomic

416 sequencing, only CSTs I, III and IV were observed (Heatmap, Fig. 5). CST II which is defined by

417 a predominance of $L$. gasseri was not identified by either sequencing technique. This might be due

418 to the small sampling size. L. fornicalis was identified in CST IV in low abundance. CST IV was

419 the most common microbial structure for Afro-Caribbean women, and was dominated by

420 Prevotella species (P. timenosis, P. amnii, P. histolitica, P. bivia, P. ihumii and P. bergensis).

421 Other genera of lower abundance within this community state type were Gardnerella, Sneathia,

422 Megasphaera and Anaerococcus (Figs. 2 and 3). This finding is in accordance with previous 
423 studies that show CST IV to be diverse and more common in African Americans [17, 20, 51, 52].

424 In general, based on both sequencing techniques used, the relative abundances of Prevotella, 425 Lactobacillus and Gardnerella dominating the cervicovaginal microbiomes of Afro-Caribbean 426 women were $23.06 \%, 22.26 \%, 10 \%$ and $13.91 \%, 9.37 \%, 12.14 \%$ for $16 \mathrm{Sr}$ RNA V4 and WGS, 427 respectively. The high abundance of Prevotella found in the Afro-Caribbean cohort has also been

428 reported for African American women though not as the dominant genus [17]. In contrast, native 429 West African women have been noted to have very low abundances (0.08-1.2\%) of Prevotella 430 [16]. Given that both Afro-Caribbean and African American women are descendants of West 431 Africans, one might have expected these ethnic groups to have similar abundances of Prevotella. 432 However, this was not the case among this Afro-Caribbean cohort. Therefore, in addition to genetic 433 factors [53], it is likely that cervicovaginal microbial communities are also influenced by 434 geophysical environments and sub-culture factors acting upon host gene expression (analogous to 435 the impact of diet on foetal/neonatal gut microbiome development) $[54,55]$. Based on our results, 436 the highly diverse cervicovaginal microbiome of the Afro-Caribbean women is a reservoir of 437 antibiotic resistance genes. Discovery of AMR genes to antibiotics such as azithromycin (marketed 438 as Zithromax $\left.{ }^{\mathrm{TM}}\right)$, Clindamycin $\left(\mathrm{Cleocin}^{\mathrm{TM}}\right)$ and nitroimidazoles (metronidazole/Flagyl $\left.{ }^{\mathrm{TM}}\right)$ in this 439 resistome is significant as these drugs are the mainstay of treatment of many common STIs and 440 BV. As is documented in literature, black women with predominantly CST IV cervicovaginal 441 bacterial communities have a greater prevalence of $\mathrm{BV}$ which is associated with increased risk of 442 STIs, HIV and HPV acquisition [13, 37, 56].

443 Immuno-Munibiome Model: a conceptual hypothesis of cervicovaginal microbiome 444 structure and regulation 
445 The regulatory mechanism involving CST and vaginal eubiosis is not well understood. The 446 absence or dominance of specific planktonic prokaryotes such as Lactobacilli or a core 447 microbiome do not completely account for vaginal health. Our findings, along with the analysis of 448 referenced vaginal microbiome studies, suggest that emphasis should perhaps be placed more on 449 microbial functions rather than on the abundance of a specific bacterial genus comprising vaginal 450 microbiomes. Thus a "core microbiome" should fundamentally be thought of as a "munibiome" 451 (termed by authors, Latin; muni "functions", biome "bacterial community") where the function(s) 452 of a group of organisms assumes greater relevance.

453 Here, based in part on the findings of this study, we introduce a hypothetical concept, the immuno454 munibiome model, for the regulation of the vaginal ecosystem in the Afro-Caribbean cohort. We 455 posit that vaginal eubiosis is maintained by two opposing microbial states. The first being a 456 facultative anaerobic state (FAS) governed by lactic acid producing microbes (such as 457 Lactobacillus and Bifidobacterium species) and the second an obligate anaerobic state (OAS) 458 governed by species (such as Prevotella and Gardnerella) that produce biogenic amines (Figs. 6 459 and 7). We suggest that one anaerobic state is more dominant than the other. Based on our research 460 findings, the cervicovaginal microbiome CST IV (with Prevotella having the highest relative 461 abundance) was the dominant community state type for our Afro-Caribbean cohort. Therefore, for 462 this group we considered the OAS to be the dominant state; one that is likely heritable. It has been 463 suggested that host genetics may in fact be a determining factor in defining the community state 464 types among females of different ethnicities [57]. In addition, heritability estimates determined by 465 variance component methods using Sequential Oligogenic Linkage Analysis Routines (SOLAR) 466 in a recent study involving 542 Korean women showed Prevotella to be the most heritable (72.2\%) 467 taxa within the vaginal microbiota of women with BV. For the Lactobacillus species, L. crispatus 
and $L$. iners had heritable estimates of $36.9 \%$ and $41.2 \%$, respectively [51]. Interestingly, CST IV has been described in the literature as asymptomatic BV, albeit an ill-defined purported disease [58].

In this study, there were increased biological processes related to bacterial-type flagellumdependent cell motility, cell adhesion, response to biotic stimulus and quorum sensing. These processes are known to facilitate biofilm formation $[11,59,60]$. Curiously, sporulation was also noted to be upregulated. Sporulation is probably involved in vaginal biofilm development since mutations in spoOA genes that encode major early sporulation transcription factor required for sporulation have been found to inhibit biofilm formation in Bacillus subtilis [61]. Additionally, fragile wall-less planktonic mycoplasmas like M. hominis ATCC 23114 and U. urealyticum identified in this research are not expected to survive the hostile vaginal environment and so it is inferred that these and other pathobionts are protected by their integration into vaginal biofilms: dynamic, highly organized adherent bacterial communities self-encased in an extracellular polysaccharide (EPS) that forms a matrix. Within the matrix, microbial interactions and controlled differential gene expression to extracellular stimuli and other vaginal bacteria are facilitated by quorum sensing and signalling. Switching between the planktonic and complex multicellular modes is also regulated by chemical signalling $[11,12,62]$.

It is known that G. vaginalis can strive in the vagina in the presence of high concentrations of lactic acid, hydrogen peroxide, bactericidins and antibiotics due to the formation of protective biofilms [63]. G. vaginalis biofilms, enhanced by Prevotella, harbour a plethora of obligate anaerobes (such as Mycoplasma, Sneathia, Gemella, Mobiluncus, Atopobium and Megasphaera amongst other anaerobes) $[64,65]$. Some species or strains of Gardnerella do not cause disease $[11,14,66]$. In this research, G. vaginalis 409-05 was more abundant than G. vaginalis ATCC 
49114019 among this group of asymptomatic women (Fig. 5 and Supplementary Table S5).

492 Intriguingly, the genome of G. vaginalis 409-05 lacks several mucin degrading and antibiotic

493 resistance genes (important virulence factors) compared to G. vaginalis ATCC 14019 [67]. Like

494 Prevotella and Gardnerella species, Lactobacillus jensenii has been shown to form vaginal 495 biofilms in vivo $[12,68,69]$. Therefore, we further propose that the two opposing anaerobic states 496 (FAS and OAS) exist as dynamic vaginal biofilms. We envision that metabolic activities within 497 these anaerobic states induce local vaginal anti-inflammatory $\left(\mathrm{I}_{\mathrm{A}}\right)$ and pro-inflammatory $\left(\mathrm{I}_{\mathrm{P}}\right)$ 498 responses that once in equilibrium maintain vaginal health.

499 Biogenic amines (e.g. putrescine, cadaverine, phenethylamine, tyramine) synthesized by obligate 500 anaerobes (e.g. Prevotella and Gardnerella), Gram-negative microbial lipopolysaccharides (LPS) 501 and other bacterial products (lipoproteins/lipopeptides) within OAS induce pro-inflammatory 502 responses via TLR-4 and NF- $\kappa$ B resulting in the release of cytokines and chemokines: an increase 503 in IL-1a, IL-1 $\beta$, IL-6, IL-8, TNF- $\alpha$, IFN- $\gamma$, RANTES and a decrease in Lipocalin-2 (Neutrophil 504 gelatinase-associated lipocalin, NGAL) secreted by neutrophils $[13,70,71,72,73,74]$. In this 505 hypothesis, the pro-inflammatory response is mitigated by an anti-inflammatory immune response 506 triggered within the FAS by lactic acid produced by Lactobacillus and other LABs (e.g. 507 Bifidobacterium, Corynebacterium, Aerococcus, Enterococcus and Atopobium vaginae), in which 508 IL-6, IL-8, TNF- $\alpha$, CCL5 (a chemotactic cytokine) and macrophage inflammatory protein, MIP$5093 \alpha$ are inhibited $[75,76,77]$. Vaginal epithelial cells secrete less than $15 \%$ of L-lactic acid via 510 anaerobic metabolism of glycogen. Lactobacillus species produce and regulate the majority of D 511 and L enantiomers of lactic acid within the vaginal mucus [8]. This lactic acid is generated from 512 the hydrolysis of maltose, maltotriose, maltotetraose and $\alpha$-dextrins which are products of cell-free 513 vaginal glycogen catabolized by $\alpha$-amylase. The latter is secreted by endocervical and fallopian 
514 tube epithelial cells [78]. The quantity of vaginal cell-free glycogen is not significantly correlated

515 with oestrogen levels [79]. However, high levels of cell-free glycogen positively correlate with a

516 high abundancy of L. crispatus and L. jensenii, but not L. iners [80, 81]. Free glycogen is released

517 from vaginal epithelial cells via cell lysis and epithelial cell membrane perforation by the action

518 of matrix metalloproteinase-8 (MMP-8) hyaluronidase-1 and cytolysin generated by Lactobacillus

519 [9]. At pH 3.86, 50\% of the lactic acid is in equilibrium with lactate and hydrogen ions. Uptake of 520 hydrogen ions by obligate anaerobes are utilized in decarboxylation of amino acids within the

521 bacterial cytoplasm to produce biogenic amines. The release of the biogenic amines elevates the

$522 \mathrm{pH}(>4.5)$ within the vaginal ecosystem inhibiting Lactobacillus growth [71].

523 A pro-inflammatory response is associated with a high $\mathrm{pH}$ which is unfavourable for facultative 524 anaerobes. Under these circumstances, perhaps through quorum sensing, lactate dehydrogenase is 525 upregulated and L. iners (presumably along with other non-lactobacillus LABs) which is better 526 adapted (than L. crispatus, L. gasseri and L. jensenii) to this environment proliferates to become 527 dominant (CST III) [82]. The resulting increase in L-lactic acid production effectively reduces the $528 \mathrm{pH}$, increases release of intracellular glycogen via pore-forming cholesterol-dependent cytolysin, 529 inerolysin [83] shifting the $\mathrm{I}_{\mathrm{A}}-\mathrm{I}_{\mathrm{P}}$ equilibrium leftwards. We postulate that with this equilibrium 530 shift (lower $\mathrm{pH}$ and higher cell-free glycogen concentrations) that either L. crispatus, L. gasseri or 531 L. jensenii which are more efficient at producing the more protective D-lactic acid [84] out 532 compete other species, with the most adaptable species (and hence CSTs I, II or V) becoming 533 dominant. Similar to the OAS biofilm, various Lactobacilli species are expected to co-exist in 534 LAB biofilm of the FAS. In essence, we believe that the cervicovaginal microbiome cycles through 535 various Lactobacillus-dominant CSTs depending on the local vaginal environment and external 536 influences (e.g. contraceptive use, antibiotic therapy, coitus, douching, menstruation). We, 
537 therefore, speculate that CSTs I, II, III and V are snapshots of an active facultative anaerobic state.

538 In fact, the temporal dynamics of these transient CSTs at the individual level have been well

539 documented [20, 45, 85].

540 In this model, only the FAS (dominant in Caucasians) is influenced by oestrogen levels. Therefore,

541 any factors (such as age, obesity/ body mass index (BMI) and ethnicity) that influence oestrogen

542 levels should not have any significant impact on the OAS. Hence, as predicted, in contrast to white

543 women no correlations have been demonstrated to date between oestrogen levels, body mass index

544 (BMI) and the cervicovaginal microbiome in black women $[86,87]$ even though they possess

545 higher levels of oestrogen compared to Caucasians [88].

546 Conclusions

547 This is the first study to characterize the cervicovaginal microbiome in a cohort of Afro-Caribbean

548 women. Functional profiling of the cervicovaginal microbial communities suggested the 549 occurrence of biofilm activity in this cohort. High abundances of Prevotella species and the less 550 virulent G. vaginalis 409-05 strain in the cervicovaginal microbiomes of these women (that were 551 clinically asymptomatic of vaginal infection), along with published discoveries of vaginal 552 Lactobacillus biofilms point towards a possible protective role of (facultative and obligate 553 anaerobe) biofilms in vaginal health. The conceptualized immuno-munibiome model (based on 554 inflammatory responses controlled by metabolic activity within biofilms of opposing obligate and 555 facultative anaerobic states) accounts for the CSTs and regulation of this vaginal ecosystem. In 556 addition, expression of macrolide-lincosamide streptogramin B and nitroimidazole AMR genes 557 within the cervicovaginal microbiome in this ethnic group may reduce the effectiveness of 558 azithromycin, clindamycin and metronidazole currently used to treat STIs and BV in Afro- 
559 Caribbean women. Future studies focusing on microbial ecophysiology, biofilm activity and 560 bacterial-induced inflammatory responses at the species-strain level could further contribute to our 561 understanding of the structure and regulation of the cervicovaginal microbiome.

\section{Declarations}

\section{Ethics approval and consent to participate}

564 The research protocol of this study was approved (IRB\# 170710-B) by the Research Ethics 565 Committee (REC) and Institutional Review Board (IRB) of The University of the West Indies, 566 Cave Hill Campus, Barbados. Written informed consent was obtained from all participating 567 volunteers.

\section{Consent for publication}

569 Not applicable

\section{Availability of data and materials}

571 The nucleotide sequence data for 16S rRNA V4 and WGS metagenomics for this study were

572 deposited in the European Nucleotide Archive (ENA) and MGnify at EMBL-EBI under accession 573 numbers PRJEB34744 (MGYS00005125) and PRJEB34967 (MGYS00005124), respectively.

574 Data are also available on MG-RAST server: mgp89180 and mgp88579.

\section{Competing Interests}

576 The authors declare no competing interests.

\section{$577 \quad$ Funding}


578 This research was financially supported by the UWI Graduate Student Research Award 579 Committee.

580 Author Contributions

581 In this study OR conceptualized, designed, performed laboratory work, bioinformatic analyses

582 and wrote the manuscript. CK prepared the libraries and MT performed Illumina next-generation 583 sequencing on samples. AA, KN and MT critically reviewed the manuscript.

584 Acknowledgments

585 The authors are grateful for necessary facilities provided by the Head, Department of Biological 586 and Chemical Sciences, University of the West Indies, Cave Hill Campus. We thank Dr. Bidyut 587 Mohapatra for his expert advice and all volunteers who participated in this research.

\section{Supporting Information}

589 Table S1. Sequence statistics of 15 cervicovaginal samples from Afro-Caribbean women (Whole 590 genome shotgun metagenomics). (MS)

591 Table S2. OTU statistics of 18 cervicovaginal samples from Afro-Caribbean women (16S rRNA 592 V4 sequencing). (MS)

593 Table S3. Relative abundance values and operational taxonomic units (OTUs) derived from partial 594 16S rRNA V4 sequences for cervicovaginal microbiota in Afro-Caribbean women (sheet 1A). The 595 identified taxa based on OTUs sequences and raw abundances are listed in sheet 1B. Color codes 596 are used to represent the species niche or conflicts: Red= conflicts, non-bacteria, non-human; 597 Blue $=$ gut and oral microbiota; Green $=$ Soil or plant microbiota. $($ XLSX) 
Table S4. Results of taxa obtained from blasting OTU sequences (from 16S rRNA V4 sequencing) using SeqMatch version 3 in the online Ribosomal Database Project (RDP: release 11_5) (Cole et al., 2014). (PDF). Available at https://data.mendeley.com/datasets/gn5jgvgd4s/draft?a=0e91656e-

601

602

603

604

605

606

607

608

609

610

611

612

613

614

615

616

617 and unweighted UniFrac. Beta diversity was visualized using 3D principal coordinates analysis

618 (PCoA). (MS)

f307-4574-94de-b448741b14b2

Table S5. Relative abundance values for all taxa in cervicovaginal samples from Afro-Caribbean women based on WGS sequencing (Sheet 1A). Relative abundance values for top 61 taxa in cervicovaginal samples from Afro-Caribbean women (Sheet 1B). The mean combined abundances for top 30 genera and species/strain are shown for all Afro-Caribbean women (Sheet 1C). (XLSX)

Table S6. Most abundant taxa for cervicovaginal microbiota in Afro-Caribbean women based on $16 \mathrm{~S}$ rRNA V4 sequencing and whole genome shotgun metagenomics. Results for the most abundant taxa based on WGS sequencing are summarized in Table 1. (XLSX)

Table S7. Metadata for 18 Afro-Caribbean women

Table S8. Statistical analysis of differential abundance of three most abundant taxa and Mycoplasma. (PDF)

Table S9. Antibiotic resistance genes in the cervicovaginal microbiomes of Afro-Caribbean women. (XLSX)

Figure S1. Alpha and Beta diversity metrics used for 16S rRNA V4 sequencing and whole genome shotgun metagenomics. Metrics for alpha diversity: Chao-1 bias-corrected, Shannon entropy and Simpson's index. Metrics for beta diversity: Bray-Curtis, Jaccard, Euclidean, weighted UniFrac
(PCA). (MS) 
619 Figure S2. Heatmap showing the relative abundance of a set of 98 functional profiles for the 620 cervicovaginal microbiome of Afro-Caribbean women based on whole genome shotgun 621 metagenomic sequence analysis.

622 References

623 1. Crosby DA, Feehily C, Cabrera-Rubio R, Higgins S, Cotter P, McAuliffe F. 541: The 624 vaginal microbiota differs between women who deliver preterm relative to those who 625 deliver fullterm. Am J Obstet Gynecol. 2019;220 Suppl 1. S361

626 2. Laven J, Koedooder R, Blockeel C, Mackens S, Budding A, Fares D, et al. Identification 627 and evaluation of the microbiome in the female and male reproductive tracts. Hum Reprod 628 Update. 2019;25(3):298-325

629 3. Racicot K, Cardenas I, Wünsche V, Aldo P, Guller S, Means RE, et al. Viral infection of 630 631 the pregnant cervix predisposes to ascending bacterial infection. $\mathrm{J}$ Immunol. 2013;191(2):934-41

632 4. Cumming HE, Bourke NM. Type I IFNs in the female reproductive tract: The first line of 633 defense in an ever-changing battleground. J Leukoc Biol. 2019;105(2):353-61.

634 5. Shey MS, Maharaj N, Archary D, Ngcapu S, Garrett N, Abdool Karim S, et al. Modulation 635 of female genital tract-derived dendritic cell migration and activation in response to 636 inflammatory cytokines and toll-like receptor agonists. PLoS One. 2016;11(5):1-15.

637 6. Wolf AJ, Underhill DM. Peptidoglycan recognition by the innate immune system. Nat Rev 638 Immunol. 2018; 18(4):243-54.

639 7. Miller EA, Beasley DAE, Dunn RR, Archie EA. Lactobacilli dominance and vaginal pH: 
Why is the human vaginal microbiome unique? Front Microbiol. 2016;7:1-13.

641 8. Boskey ER, Cone RA, Whaley KJ, Moench TR. Origins of vaginal acidity: High D/L lactate 642 ratio is consistent with bacteria being the primary source. Hum Reprod. 2001;16(9):1809643 13.

9. Tachedjian G, Aldunate M, Bradshaw CS, Cone RA. The role of lactic acid production by 645 probiotic Lactobacillus species in vaginal health. Res Microbiol. 2017;168:782-92.

646

647

648

649

650

651

652

653

654

655

656

657

658

659

660

10. Baron SA, Diene SM, Rolain JM. Human microbiomes and antibiotic resistance. Hum Microbiome J. 2018; 10:43-52.

11. Campisciano G, Zanotta N, Petix V, Corich L, De Seta F, Comar M. Vaginal microbiota dysmicrobism and role of biofilm-forming bacteria. Front Bios. 2018;10:528-536.

12. Hardy L, Cerca N, Jespers V, Vaneechoutte M, Crucitti T. Bacterial biofilms in the vagina. Res Microbiol. 2017;168:865-74.

13. Anahtar MN, Byrne EH, Doherty KE, Bowman BA, Yamamoto HS, Soumillon M, et al. Cervicovaginal bacteria are a major modulator of host inflammatory responses in the female genital tract. Immunity. 2015; 42(5):965-76.

14. Hickey RJ, Forney LJ. Gardnerella vaginalis does not always cause Bacterial Vaginosis . J Infect Dis. 2014; 210(10):1682-3.

15. Larsen B, Monif GRG. Understanding the Bacterial Flora of the Female Genital Tract. Clin Infect Dis. 2001; 32(4):e69-77.

16. Okoli AC, Agbakoba NR, Ezeanya CC, Oguejiofor CB, Anukam KC. Comparative abundance and functional biomarkers of the vaginal and gut microbiome of Nigerian 
women with bacterial vaginosis : A Study with 16S rRNA Metagenomics. J Med Lab Sci.

662 2019;29(1):1-26.

663 17. Ravel J, Gajer P, Davis CC, Peralta L, McCulle SL, Forney LJ, et al. Vaginal microbiome 664 of reproductive-age women. Proc Natl Acad Sci. 2010;108(Supplement_1):4680-7.

665 18. Puri S. Cytolytic vaginosis: A common yet under-diagnosed entity. J Cytol Histol. 666 2019;10(6):10-3.

667 19. DiGiulio DB, Callahan BJ, McMurdie PJ, Costello EK, Lyell DJ, Robaczewska A, et al. 668 Temporal and spatial variation of the human microbiota during pregnancy. Proc Natl Acad 669 Sci. 2015;112(35):11060-5.

670 20. Gajer P, Brotman RM, Bai G, Sakamoto J, Schütte UME, Zhong X, et al. Temporal 671 dynamics of the human vaginal microbiota. Sci Transl Med. 2012;4(132):132ra52.

672 21. Ata B, Yildiz S, Turkgeldi E, Brocal VP, Dinleyici EC, Moya A, et al. The Endobiota Study: 673 Comparison of Vaginal, Cervical and Gut Microbiota Between Women with Stage 3/4 674 Endometriosis and Healthy Controls. Sci Rep. 2019;9(1):1-9.

675 22. Comar M, De Seta F, Zanotta N, Licastro D, Campisciano G. In vivo microbiome and 676 associated immune markers: New insights into the pathogenesis of vaginal dysbiosis. Sci 677 Rep. 2018;8(1):1-9.

678 23. Hong KH, KukHong S, Sung ImCho MT, EunkyungRa MT, Han KH, Kang SB, et al. 679 Analysis of the vaginal microbiome by next-generation sequencing and evaluation of its 680 performance as a clinical diagnostic tool in vaginitis. Ann Lab Med. 2016;36(5):441-9.

681 24. Virtanen S, Kalliala I, Nieminen P, Salonen A. Comparative analysis of vaginal microbiota 
sampling using 16S rRNA gene analysis. PLoS One. 2017;12(7):e0181477.

683

684

685

686

687

688

689

690

691

692

693

694

695

696

697

698

699

700

701

702

25. Virtanen S, Rantsi T, Virtanen A, Kervinen K, Nieminen P, Kalliala I, et al. Vaginal Microbiota Composition Correlates Between Pap Smear Microscopy and Next Generation Sequencing and Associates to Socioeconomic Status. Sci Rep. 2019;9(1):7750.

26. Caporaso JG, Lauber CL, Walters WA, Berg-Lyons D, Lozupone CA, Turnbaugh PJ, et al. Global patterns of $16 \mathrm{~S}$ rRNA diversity at a depth of millions of sequences per sample. Proc Natl Acad Sci U S A. 2011;108 Suppl: 4516-22.

27. Moustafa A, Li W, Singh H, Moncera KJ, Torralba MG, Yu Y, et al. Microbial metagenome of urinary tract infection. Sci Rep. 2018;8(1):4333.

28. Andrews S. FastQC A Quality Control tool for High Throughput Sequence Data. 2010. http://www.bioinformatics.babraham.ac.uk/projects/fastqc/.

29. Meyer F, Bagchi S, Chaterji S, Gerlach W, Grama A, Harrison T, et al. MG-RAST version 4-lessons learned from a decade of low-budget ultra-high-throughput metagenome analysis. Brief Bioinform. 2019;20(4):1151-1159

30. Cole JR, Wang Q, Fish JA, Chai B, McGarrell DM, Sun Y, et al. Ribosomal Database Project: Data and tools for high throughput rRNA analysis. Nucleic Acids Res. 2014;42:633-42.

31. Anderson MJ. A new method for non-parametric multivariate analysis of variance. Austral Ecol. 2001;(26):32-46.

32. Ashburner M, Ball CA, Blake JA, Botstein D, Butler H, Cherry JM, et al. Gene Ontology: tool for the unification of biology. Nat Genet. 2000;25(1):25-9. 
703 33. Carbon S, Douglass E, Dunn N, Good B, Harris NL, Lewis SE, et al. The Gene Ontology Resource: 20 years and still GOing strong. Nucleic Acids Res. 2019;47: 330-8.

34. El-Gebali S, Mistry J, Bateman A, Eddy SR, Luciani A, Potter SC, et al. The Pfam protein families database in 2019. Nucleic Acids Res. 2019;47(D1):D427-32.

35. Kaminski J, Gibson MK, Franzosa EA, Segata N, Dantas G, Huttenhower C. Highspecificity targeted functional profiling in microbial communities with ShortBRED. PLoS Comput Biol. 2015;11(12):e1004557.

36. Alcock BP, Raphenya AR, Lau TTY, Tsang KK, Bouchard M, Edalatmand A, et al. CARD 2020: antibiotic resistome surveillance with the comprehensive antibiotic resistance database. Nucleic Acids Res. 2020;48: 517-25.

37. Lennard K, Dabee S, Barnabas SL, Havyarimana E, Blakney A, Jaumdally SZ, et al. Microbial composition predicts genital tract inflammation and persistent bacterial vaginosis in South African adolescent females. Infect Immun. 2018;86(1):1-18.

38. Herbst FA, Dueholm MS, Wimmer R, Nielsen PH. The proteome of Tetrasphaera elongata is adapted to changing conditions in wastewater treatment plants. Proteomes. 2019;7(2).

39. Kristiansen R, Nguyen HTT, Saunders AM, Nielsen JL, Wimmer R, Le VQ, et al. A metabolic model for members of the genus Tetrasphaera involved in enhanced biological phosphorus removal. ISME J. 2013;7(3):543-54.

40. Osman S, Moissl C, Hosoya N, Briegel A, Mayilraj S, Satomi M, et al. Tetrasphaera remsis sp. nov., isolated from the regenerative enclosed life support module simulator (REMS) air system. Int J Syst Evol Microbiol. 2007;57(12):2749-53. 
724 41. Ranjan R, Rani A, Metwally A, Mcgee HS, Perkins DL. Analysis of the microbiome:

725

726

727

728

729

730

731

732

733

734

735

736

737

738

739

740

741

742

743

744

Advantages of whole genome shotgun versus $16 \mathrm{~S}$ amplicon sequencing. Biochem Biophys Res Commun. 2016;469(4):967-77.

42. Arredondo A, Àlvarez G, Nart J, Mor C, Blanc V, León R. Detection and expression analysis of tet(B) in Streptococcus oralis. J Oral Microbiol. 2019;11(1).

43. Jeters RT, Rivera AJ, Boucek LM, Stumpf RM, Leigh SR, Salyers AA. Antibiotic resistance genes in the vaginal microbiota of primates not normally exposed to antibiotics. Microb Drug Resist. 2009;15(4):309-15.

44. Fettweis JM, Serrano MG, Brooks JP, Edwards DJ, Girerd PH, Parikh HI, et al. The vaginal microbiome and preterm birth. Nat Med. 2019;25(6):1012-21.

45. Goltsman DSA, Sun CL, Proctor DM, DiGiulio DB, Robaczewska A, Thomas BC, et al. Metagenomic analysis with strain-level resolution reveals fine-scale variation in the human pregnancy microbiome. Genome Res. 2018;28(10):1467-80.

46. Huber P, Cornejo-Castillo FM, Ferrera I, Sánchez P, Logares R, Metz S, et al. Primer Design for an Accurate View of Picocyanobacterial Community Structure by Using HighThroughput Sequencing. Kivisaar M, editor. Appl Environ Microbiol. 2019;85(7):e0265918

47. Jeraldo P, Chia N, Goldenfeld N. On the suitability of short reads of $16 \mathrm{~S}$ rRNA for phylogeny-based analyses in environmental surveys. Environ Microbiol. 2011;13(11):3000-9.

48. Vilo C, Dong Q. Evaluation of the RDP Classifier Accuracy Using 16S rRNA Gene 
Variable Regions. Metagenomics. 2012;1:1-5.

746

747

748

749

750

751

752

753

754

755

756

757

758

759

760

761

762

763

764

765
49. Atassi F, Brassart D, Grob P, Graf F, Servin AL. Lactobacillus strains isolated from the vaginal microbiota of healthy women inhibit Prevotella bivia and Gardnerella vaginalis in coculture and cell culture. FEMS Immunol Med Microbiol. 2006;48(3):424-32.

50. El Aila NA, Tency I, Saerens B, De Backer E, Cools P, dos Santos Santiago GL, et al. Strong correspondence in bacterial loads between the vagina and rectum of pregnant women. Res Microbiol. 2011;162(5):506-13.

51. Fettweis JM, Serrano MG, Huang B, Brooks JP, Glascock AL, Sheth NU, et al. An emerging Mycoplasma associated with trichomoniasis, vaginal infection and disease. PLoS One. 2014 ;9(10):e110943. doi: 10.1371/journal.pone.0110943

52. Zhou X, Brown CJ, Abdo Z, Davis CC, Hansmann MA, Joyce P, et al. Differences in the composition of vaginal microbial communities found in healthy Caucasian and black women. ISME Journal. 2007;1(2):121-33.

53. Si J, You HJ, Yu J, Sung J, Ko GP. Prevotella as a Hub for Vaginal Microbiota under the Influence of Host Genetics and Their Association with Obesity. Cell Host Microbe. 2017;21(1):97-105.

54. Gupta VK, Paul S, Dutta C. Geography, ethnicity or subsistence-specific variations in human microbiome composition and diversity. Front Microbiol. 2017;8:1162. doi: 10.3389/fmicb.2017.01162

55. Li Y. Epigenetic mechanisms link maternal diets and gut microbiome to obesity in the offspring. Front Genet. 2018;9:1-13. 
766 56. Nye MB, Harris AB, Pherson AJ, Cartwright CP. Prevalence of Mycoplasma genitalium infection in women with bacterial vaginosis. BMC Womens Health. 2020;20(1):1-5.

768

769

770

771

772

773

774

775

776

777

778

779

780

781

782

783

784

785

786

57. Zhou X, Hansmann MA, Davis CC, Suzuki H, Brown CJ, Schütte U, et al. The vaginal bacterial communities of Japanese women resemble those of women in other racial groups. FEMS Immunol Med Microbiol. 2010;58(2):169-81.

58. Kalia N, Singh J, Kaur M. Microbiota in vaginal health and pathogenesis of recurrent vulvovaginal infections: A critical review. Ann Clin Microbiol Antimicrob. 2020;19(1):119.

59. O’Toole GA, Kolter R. Flagellar and twitching motility are necessary for Pseudomonas aeruginosa biofilm development. Mol Microbiol. 1998;30(2):295-304.

60. Wood TK, González Barrios AF, Herzberg M, Lee J. Motility influences biofilm architecture in Escherichia coli. Appl Microbiol Biotechnol. 2006;72(2):361-7.

61. Hamon MA, Lazazzera BA. The sporulation transcription factor Spo0A is required for biofilm development in Bacillus subtilis. Mol Microbiol. 2001;42(5):1199-209.

62. Muzny CA, Schwebke JR. Biofilms: An underappreciated mechanism of treatment failure and recurrence in vaginal infections. Clin Infect Dis. 2015;61(4):601-6.

63. Patterson JL, Girerd PH, Karjane NW, Jefferson KK. Effect of biofilm phenotype on resistance of Gardnerella vaginalis to hydrogen peroxide and lactic acid. Am J Obstet Gynecol. 2007;197(2):170.e1-7

64. Machado A, Cerca N. Influence of biofilm formation by gardnerella vaginalis and other anaerobes on bacterial vaginosis. J Infect Dis. 2015; 212(12):1856-61. 
806

65. Castro J, Machado D, Cerca N. Unveiling the role of Gardnerella vaginalis in polymicrobial bacterial vaginosis biofilms: the impact of other vaginal pathogens living as neighbors. ISME Journal. 2019; 1306-17.

66. Castro J, Alves P, Sousa C, Cereija T, França Â, Jefferson KK, et al. Using an in-vitro biofilm model to assess the virulence potential of bacterial vaginosis or non-bacterial vaginosis Gardnerella vaginalis isolates. Sci Rep. 2015;5:1-10.

67. Yeoman CJ, Yildirim S, Thomas SM, Durkin AS, Torralba M, Sutton G, et al. Comparative genomics of Gardnerella vaginalis strains reveals substantial differences in metabolic and virulence potential. PLoS One. 2010; 5(8):e12411. doi: 10.1371/journal.pone.0012411.

68. Patterson JL, Stull-Lane A, Girerd PH, Jefferson KK. Analysis of adherence, biofilm formation and cytotoxicity suggests a greater virulence potential of Gardnerella vaginalis relative to other bacterial vaginosis associated anaerobes. Microbiology. 2010;156(2):392399.

69. Ventolini G. Vaginal lactobacillus: Biofilm formation in vivo - clinical implications. Int J Womens Health. 2015;7:243-7.

70. Chen KCS, Forsyth PS, Buchanan TM, Holmes KK. Amine content of vaginal fluid from untreated and treated patients with nonspecific vaginitis. J Clin Invest. 1979;63(5):828-35.

71. Nelson TM, Borgogna JLC, Brotman RM, Ravel J, Walk ST, Yeoman CJ. Vaginal biogenic amines: Biomarkers of bacterial vaginosis or precursors to vaginal dysbiosis? Front Physiol. $2015 ; 6: 1-15$.

72. Marconi C, Donders GG, Parada CMGL, Giraldo PC, Da Silva MG. Do Atopobium 
vaginae, Megasphaera sp. and Leptotrichia sp. change the local innate immune response and sialidase activity in bacterial vaginosis? Sex Transm Infect. 2013;89(2):167-73.

810

811

73. Roachford OSE, Nelson KE, Mohapatra BR. Comparative genomics of four Mycoplasma species of the human urogenital tract: Analysis of their core genomes and virulence genes. Int J Med Microbiol. 2017;307(8):508-20.

74. Roachford O, Nelson KE, Mohapatra BR. Virulence and molecular adaptation of human urogenital mycoplasmas: A review. Biotechnol Biotechnol Equip. 2019;33(1):1-10.

75. Hearps AC, Tyssen D, Srbinovski D, Bayigga L, Diaz DJD, Aldunate M, et al. Vaginal lactic acid elicits an anti-inflammatory response from human cervicovaginal epithelial cells and inhibits production of pro-inflammatory mediators associated with HIV acquisition. Mucosal Immunol. 2017;10(6):1480-90.

76. Joo HM, Hyun YJ, Myoung KS, Ahn YT, Lee JH, Huh CS, et al. Lactobacillus johnsonii HY7042 ameliorates Gardnerella vaginalis-induced vaginosis by killing Gardnerella vaginalis and inhibiting NF-אB activation. Int Immunopharmacol. 2011;11(11):1758-65.

77. Rizzo A, Fiorentino M, Buommino E, Donnarumma G, Losacco A, Bevilacqua N. Lactobacillus crispatus mediates anti-inflammatory cytokine interleukin-10 induction in response to Chlamydia trachomatis infection in vitro. Int $\mathrm{J}$ Med Microbiol. 2015;305(8):815-827.

78. Spear GT, French AL, Gilbert D, Zariffard MR, Mirmonsef P, Sullivan TH, et al. Human $\alpha$-amylase present in lower-genital-tract mucosal fluid processes glycogen to support vaginal colonization by Lactobacillus. J Infect Dis. 2014;210(7):1019-28. 
829 79. Mirmonsef P, Hotton AL, Gilbert D, Gioia CJ, Maric D, Hope TJ, et al. Glycogen levels in undiluted genital fluid and their relationship to vaginal $\mathrm{pH}$, estrogen, and progesterone. PLoS One. 2016;11(4):1-10.

832

80. Amabebe E, Anumba DOC. The vaginal microenvironment: The physiologic role of Lactobacilli. Front Med. 2018;5:1-11.

81. Mirmonsef P, Hotton AL, Gilbert D, Burgad D, Landay A, Weber KM, et al. Free glycogen in vaginal fluids is associated with Lactobacillus colonization and low vaginal pH. PLoS One. 2014;9(7):26-9.

82. Lopes dos Santos Santiago G, Tency I, Verstraelen H, Verhelst R, Trog M, Temmerman M, et al. Longitudinal qPCR Study of the Dynamics of L. crispatus, L. iners, A. vaginae, (Sialidase Positive) G. vaginalis, and P. bivia in the vagina. PLoS One. 2012;7(9) : e45281. doi: 10.1371/journal.pone.0045281

83. Pleckaityte M. Cholesterol-dependent cytolysins produced by vaginal bacteria: certainties and controversies. Front Cell Infect Microbiol. 2020;9:1-14.

84. Witkin SS, Mendes-Soares, M. LI, Jayaram Aswathi, J LW, Forney Larry J. Influence of vaginal bacteria and D- and L-lactic acid isomers on vaginal extracellular matrix metal. MBio. 2013;4(4):1-7.

85. Ravel J, Brotman RM, Gajer P, Ma B, Nandy M, Fadrosh DW, et al. Daily temporal dynamics of vaginal microbiota before, during and after episodes of bacterial vaginosis. Microbiom. 2013;23(4):1-6.

86. Brookheart RT, Lewis WG, Peipert JF, Lewis AL, Allsworth JE. Association between 
850

851

852

853

854

855

856

857

858

859

860

861

862

863

864

865

866

867

868

869

9

0

61

62

863

84

obesity and bacterial vaginosis as assessed by Nugent score. Am J Obstet Gynecol. 2019;220(5):476.e1-476.e11. doi: 10.1016/j.ajog.2019.01.229.

87. Nunn KL, Ridenhour BJ, Chester EM, Vitzthum VJ, Fortenberry JD, Forney LJ. Vaginal glycogen, not estradiol, is associated with vaginal bacterial community composition in black adolescent women. J Adolesc Heal. 2019;65(1):130-8.

88. Marsh EE, Shaw ND, Klingman KM, Tiamfook-Morgan TO, Yialamas MA, Sluss PM, et al. Estrogen levels are higher across the menstrual cycle in African-American women compared with Caucasian women. J Clin Endocrinol Metab. 2011;96(10):3199-206.

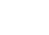


871 Fig. 1. Relative abundance of selected taxa at the species level. Microbial community structures

872 determined by 16S rRNA V4 and WGS metagenomics are similar.(A) Relative abundance of taxa

873 as determined by targeted $16 \mathrm{~S}$ rRNA V4 sequencing (B) Relative abundance of taxa as determined

874 by whole genome shotgun (relative abundances for $T$. japonica not included) (C) Relative

875 abundance of taxa as determined by whole genome shotgun (relative abundances for T. japonica

876 included). Cervicovaginal samples are labeled L100 to H210.

877 Fig. 2. (A) $16 \mathrm{~S}$ rRNA V4 sequencing data analyzed using Chao-1 bias-corrected $\alpha$-diversity

878 metric. (B) $16 \mathrm{~S}$ rRNA V4 sequencing data visualized with 3D principle coordinates analysis

879 (PCoA) for using Bray-Curtis $\beta$-diversity metrics. (C) Whole genome shotgun metagenomics data 880 visualized with 3D principal coordinates analysis (PCoA) Bray-Curtis using $\beta$-diversity metrics.

881 Refer to supplementary Figure S1 for additional analyses.

882 Fig. 3. Heatmap indicating relative abundance of bacteria in the cervicovaginal microbiome of 883 Afro-Caribbean women based on 16S rRNA V4 sequence analysis.

Fig. 4. Area chart showing relative abundance of antimicrobial (AMR) resistance genes in the 885 cervicovaginal microbiome of Afro-Caribbean women.

Fig. 5. Heatmap indicating relative abundance of bacteria in the cervicovaginal microbiome of 887 Afro-Caribbean women based on whole genome shotgun metagenomic sequence analysis.

Fig. 6. Rooted circular cladogram of taxa of cervicovaginal microbiome of Afro-Caribbean women 889 based on 16S rRNA V4 sequence analysis. Dominant facultative anaerobes (Lactobacillus species) and dominant obligate anaerobes (Prevotella species) are indicated by red and green boundaries, 891 respectively. 
892 Fig. 7. Schematic of the immune-munibiome model depicting the dynamic regulation of the 893 cervicovaginal ecosystem (pink area indicates lower vaginal tract). The left border of the diagram 894 represents the obligate anaerobe state (OAS) biofilm. On the right border is the biofilm of the 895 facultative anaerobic state. A low $\mathrm{pH}$ (high lactic acid concentration) generated by lactic acid 896 bacilli (LAB) bacteria is mitigated by the consumption of protons and production of biogenic 897 amines by obligate anaerobes. Lactic acid induces an anti-inflammatory immune response, while 898 lipopolysaccharides (LPS) and lipoproteins trigger a proinflammatory immune response. The net 899 result is equilibrium of inflammatory responses and $\mathrm{pH}$ changes that maintains vaginal eubiosis. 900 901 902 903 904 905 906 907 908 909 910 
91Fig. 1.

913
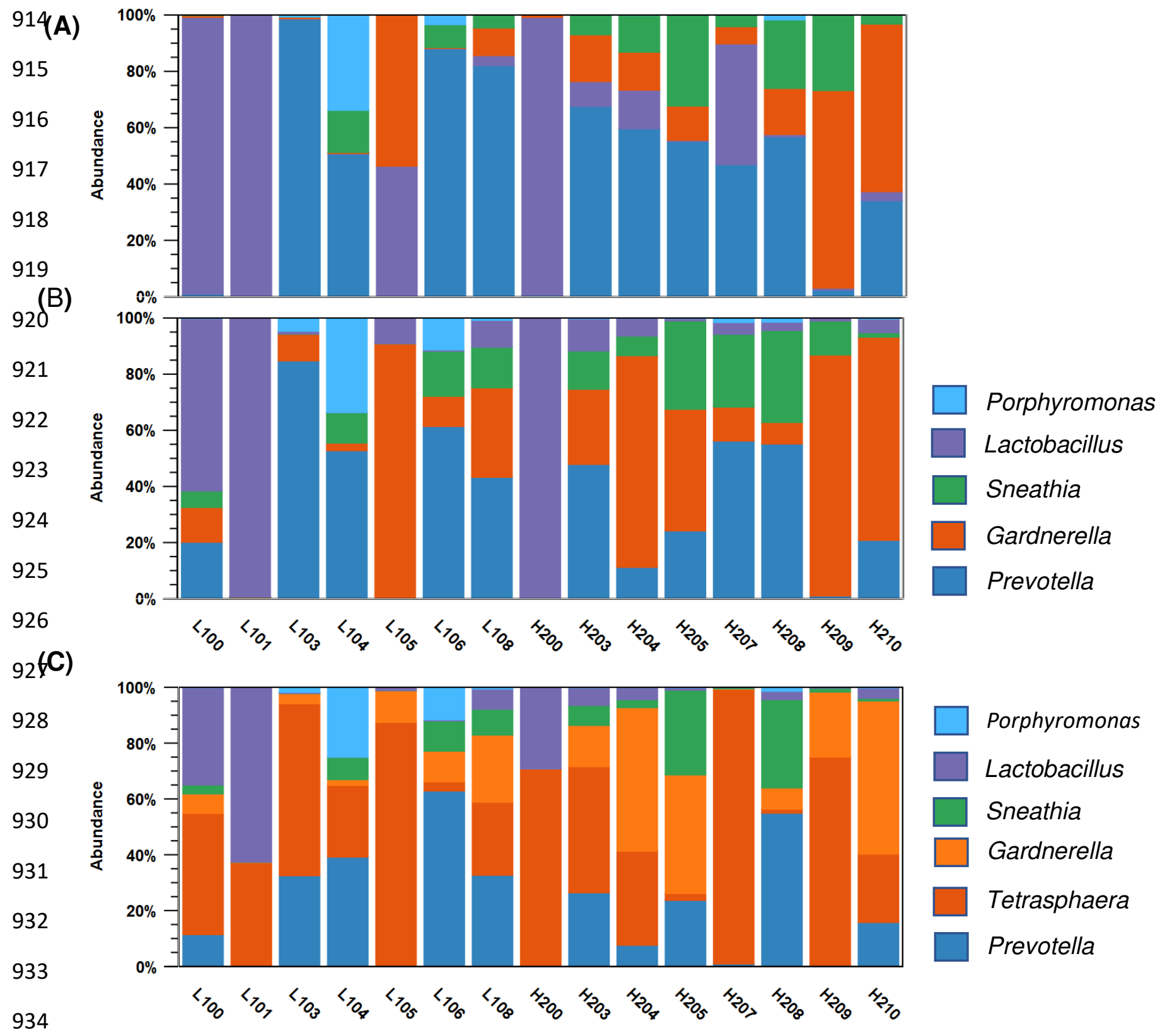

935

936

937

938 
93Fig. 2.

94ФА)

941

942

943

944

945

946

947

948

949

950

951

952

953

954

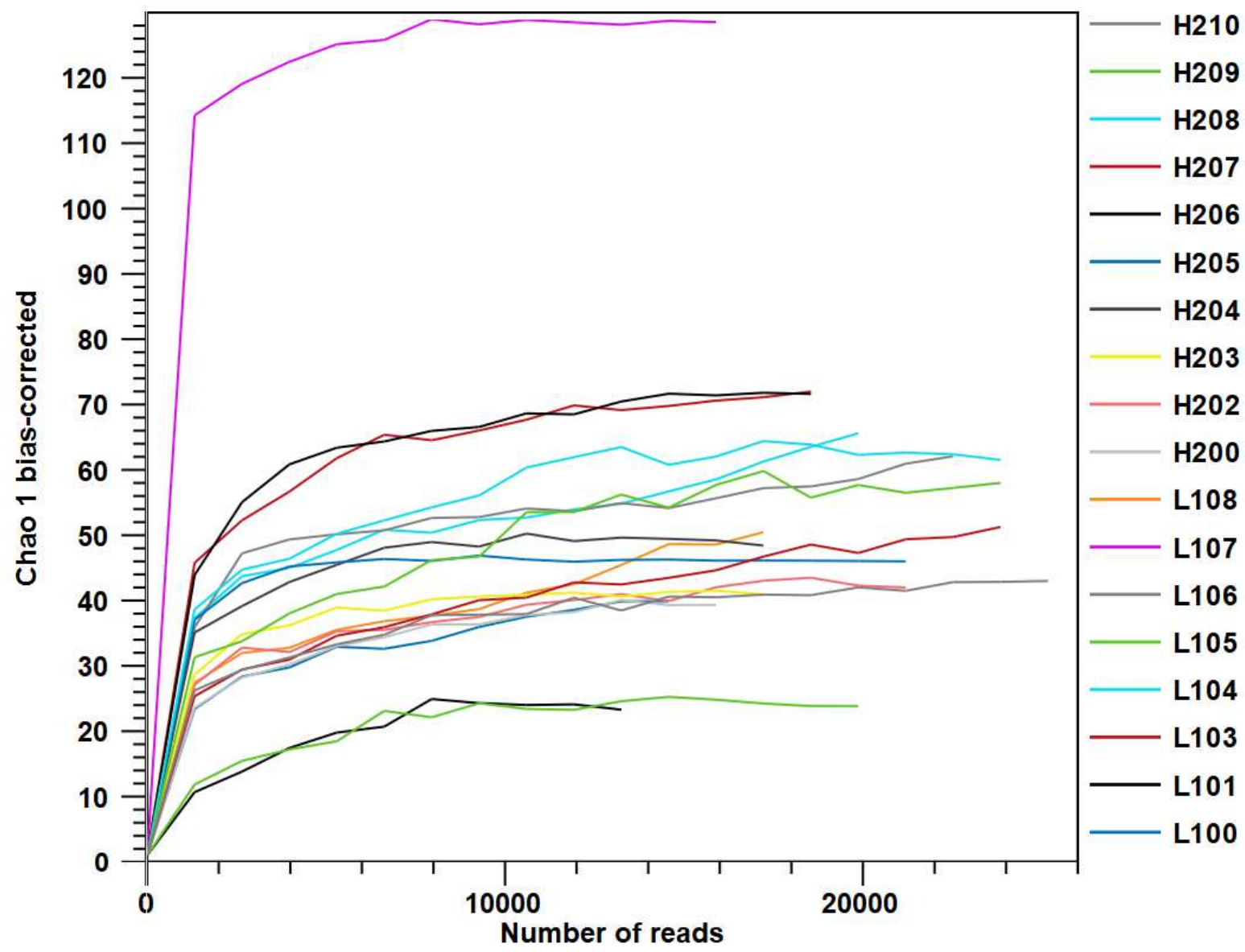

955

956 (B)

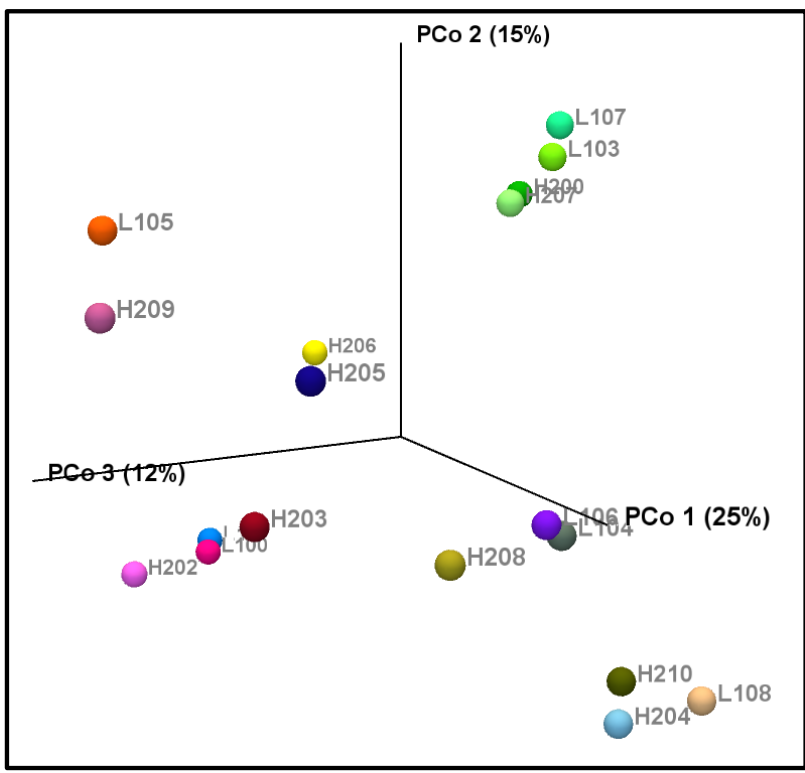

957
(C)

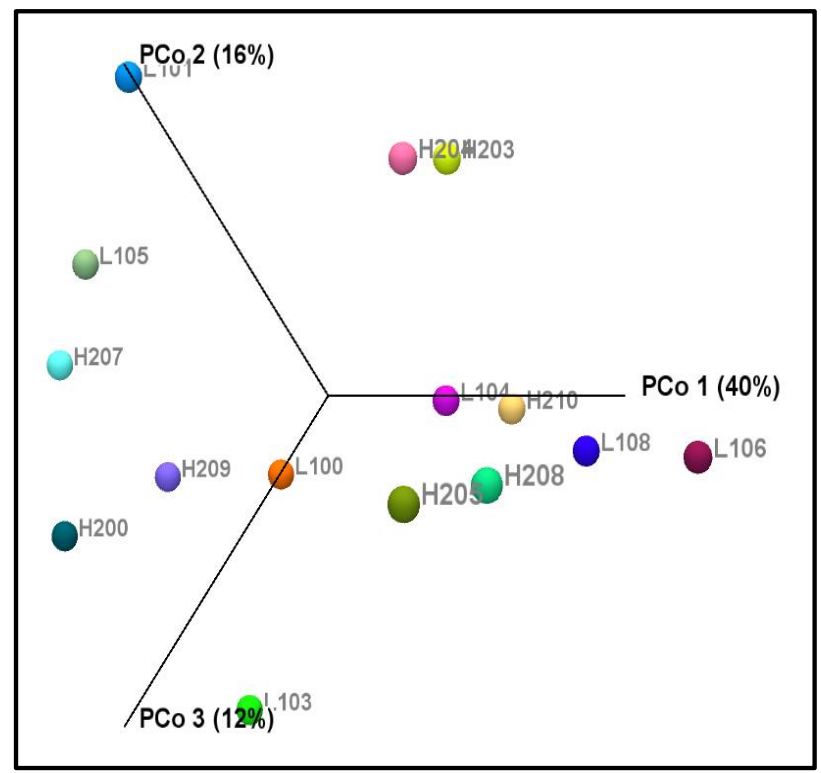


Fig. 3.

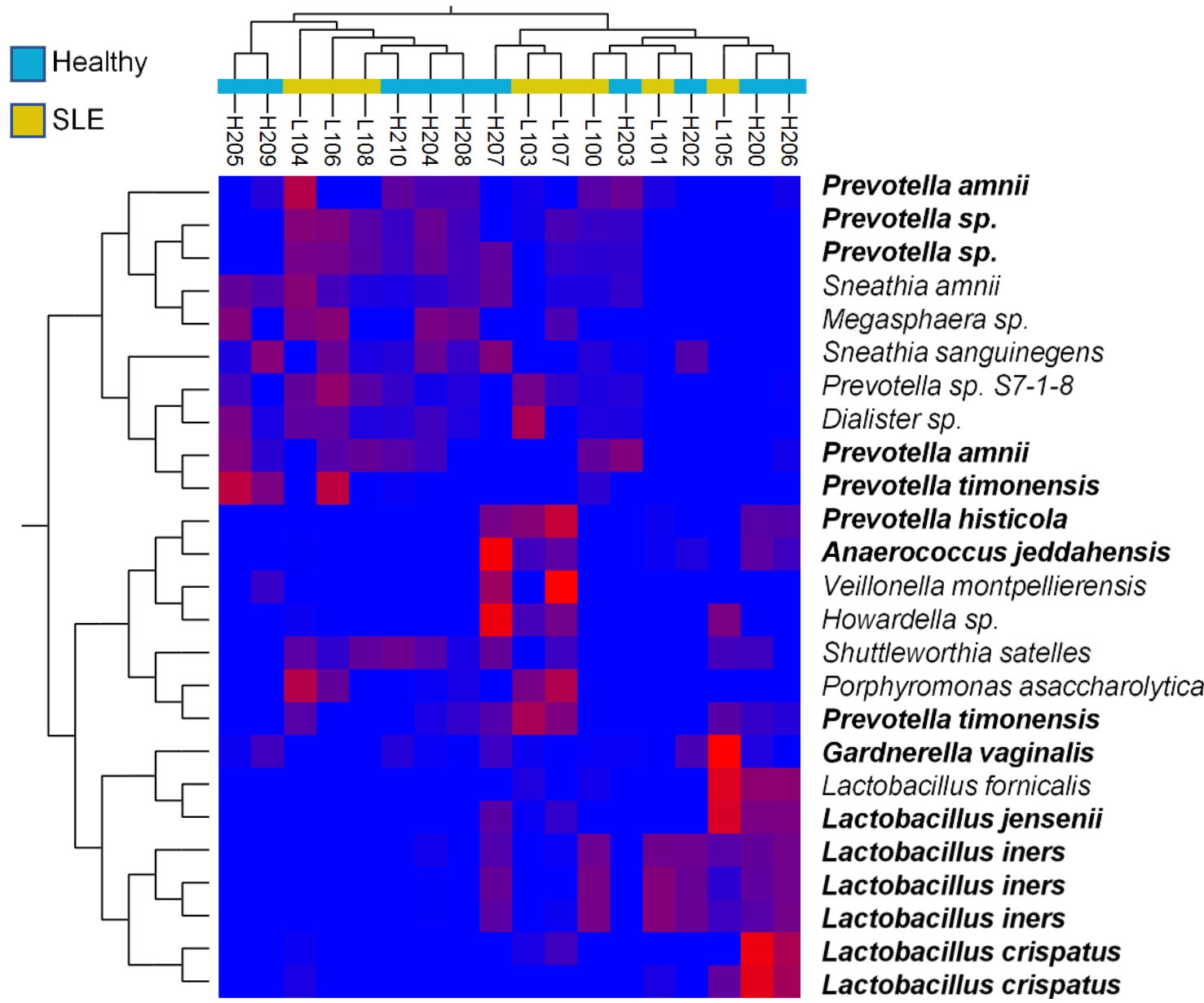

Clustering: Sample and feature clustering

Data: Normalized abundances

Dissimilarity: Euclidean distance

Linkage: Complete linkage

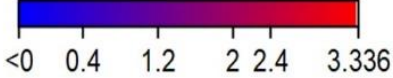

Taxon Abundance

959

960

961

962

963

964

965 
96Fig.4.

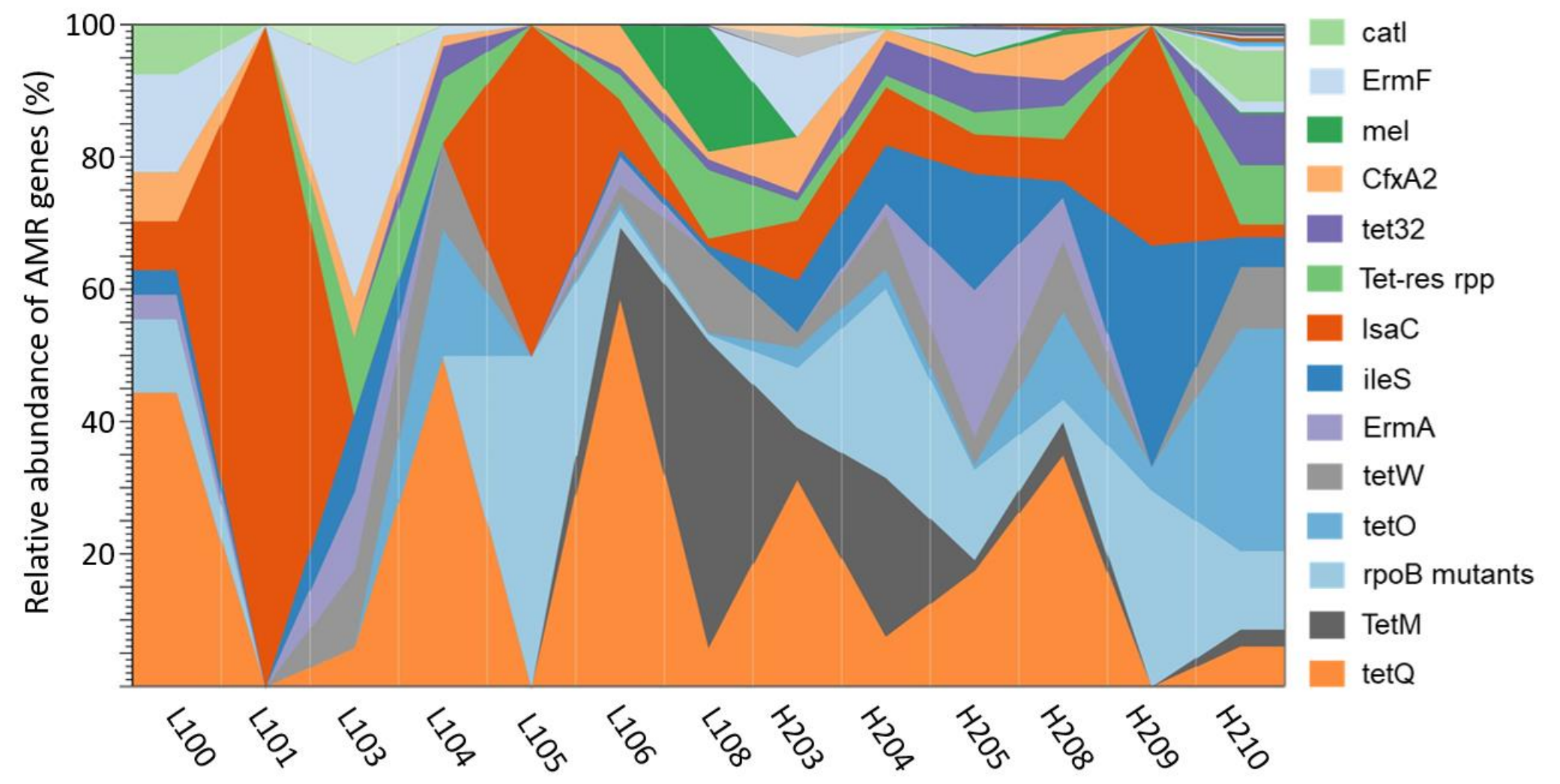

96 AMR = Antimicrobial resistance

968

969

970

971

972

973

974

975

976

977

978

979 

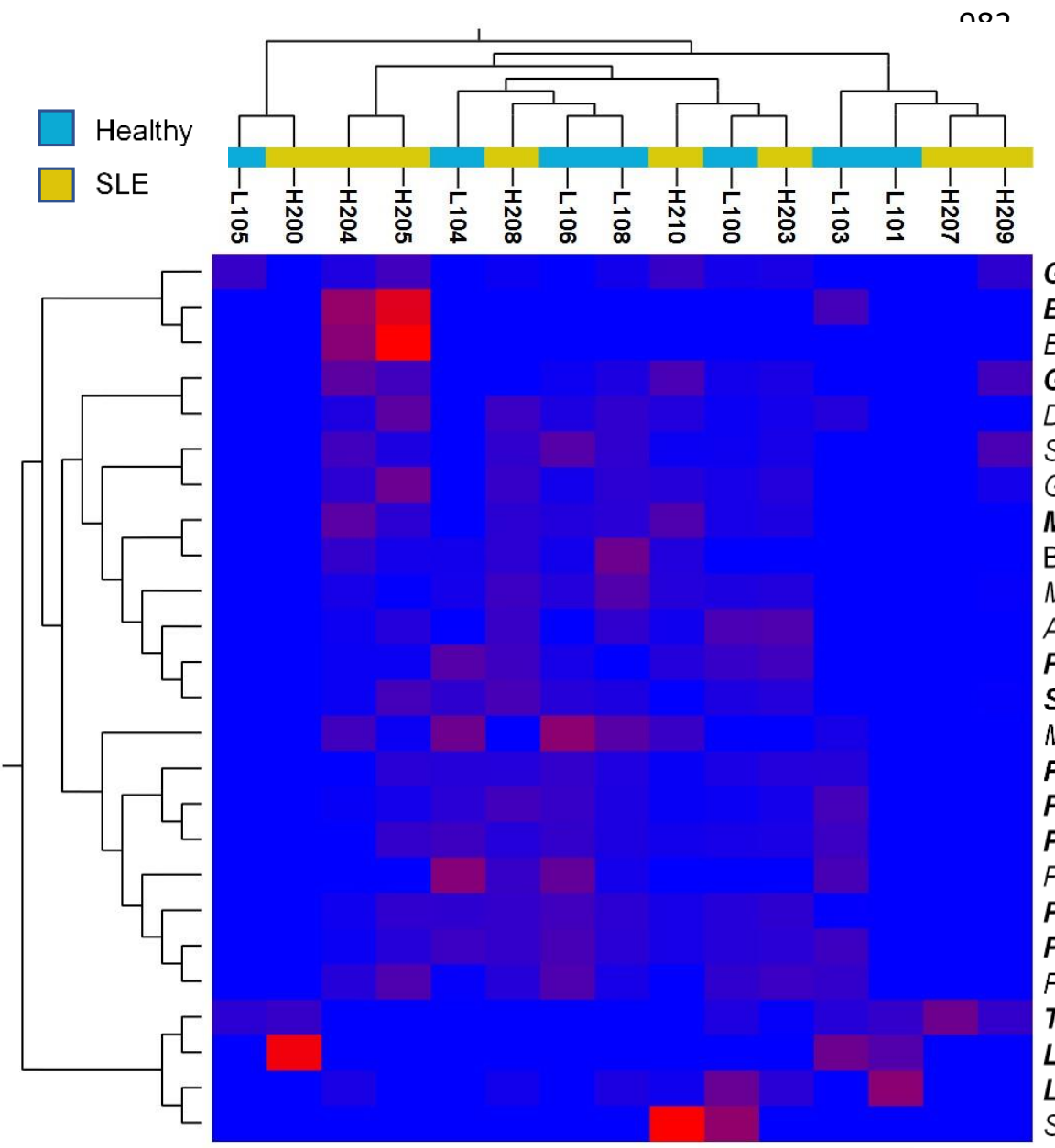

Gardnerella vaginalis ATCC 14019

Bifidobacterium breve DSM 20213

Bifidobacterium longum NCC2705

Gardnerella vaginalis 409-05

Dialister micraerophilus DSM 19965

Sneathia sanguinegens

Gamella asaccharolytica

Megasphaera genomosp. type_1 str. 28L

Bacteria; Firmicutes

Mageeibacillus indolicus UP119-5

Atopobium vaginae DSM 15829

Prevotella amnii DSM 23384

Sneathia amnii

Mobiluncus mulieris ATCC 35243

Prevotella sp.

Prevotella timonensis DSM 22865

Prevotella bivia DSM 20514

Porphyromonas asaccharolytica DSM 20707

Prevotella ihumii

Prevotella bergensis DSM 17361

Peptoniphilus lacrimalis DSM 7455

Tetrasphaera japonica $\mathrm{T} 1-\mathrm{X} 7$

Lactobacillus crispatus ST1

Lactobacillus iners DSM 13335

Staphylococcus aureus NCTC 8325

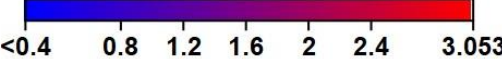

Taxon Abundance

984

Clustering: Sample and feature clustering

Data: Normalized abundances

Dissimilarity: Euclidean distance

Linkage: Complete linkage 
Fig9 6.

992

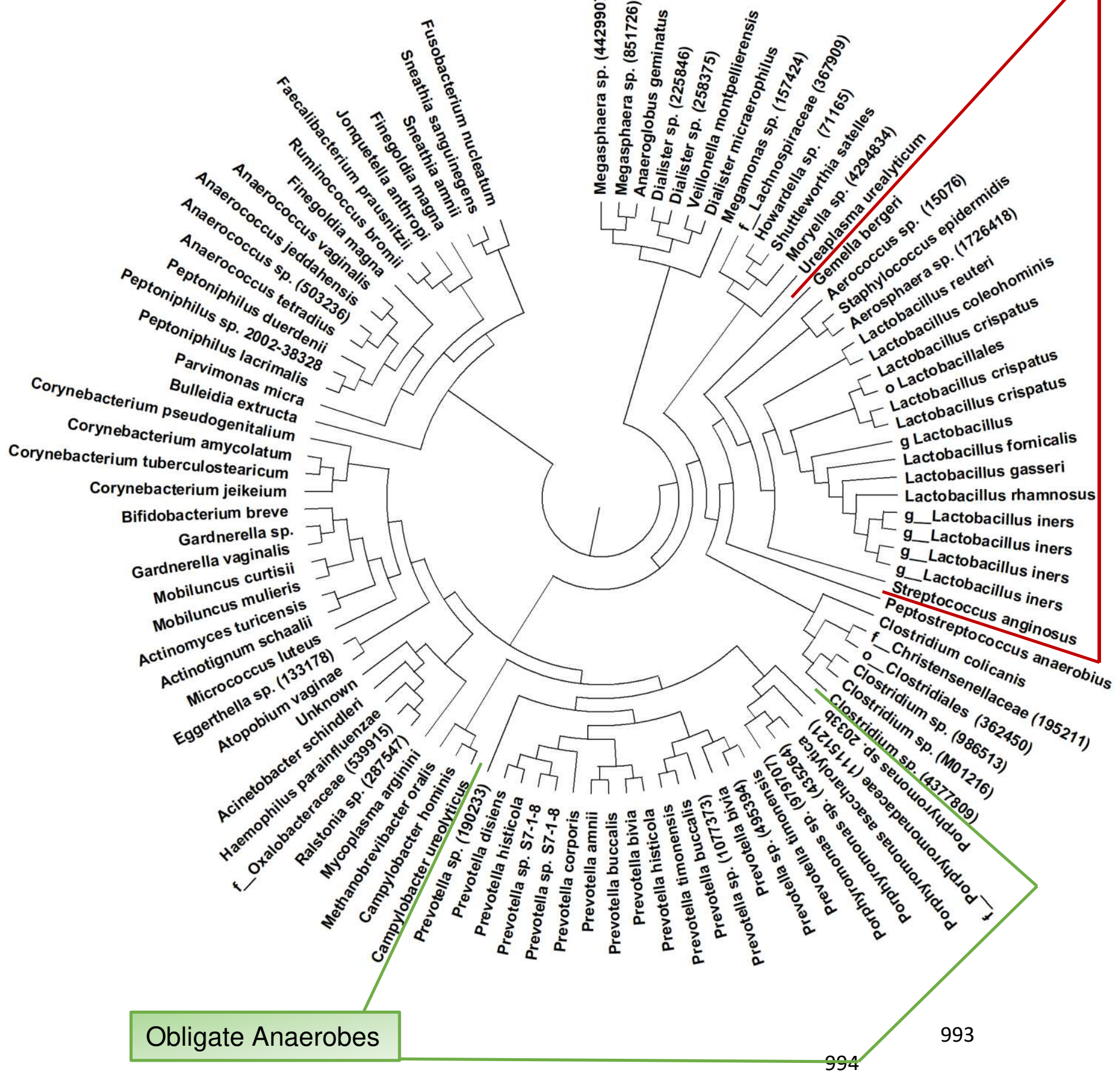


Fig. $\$ 97$

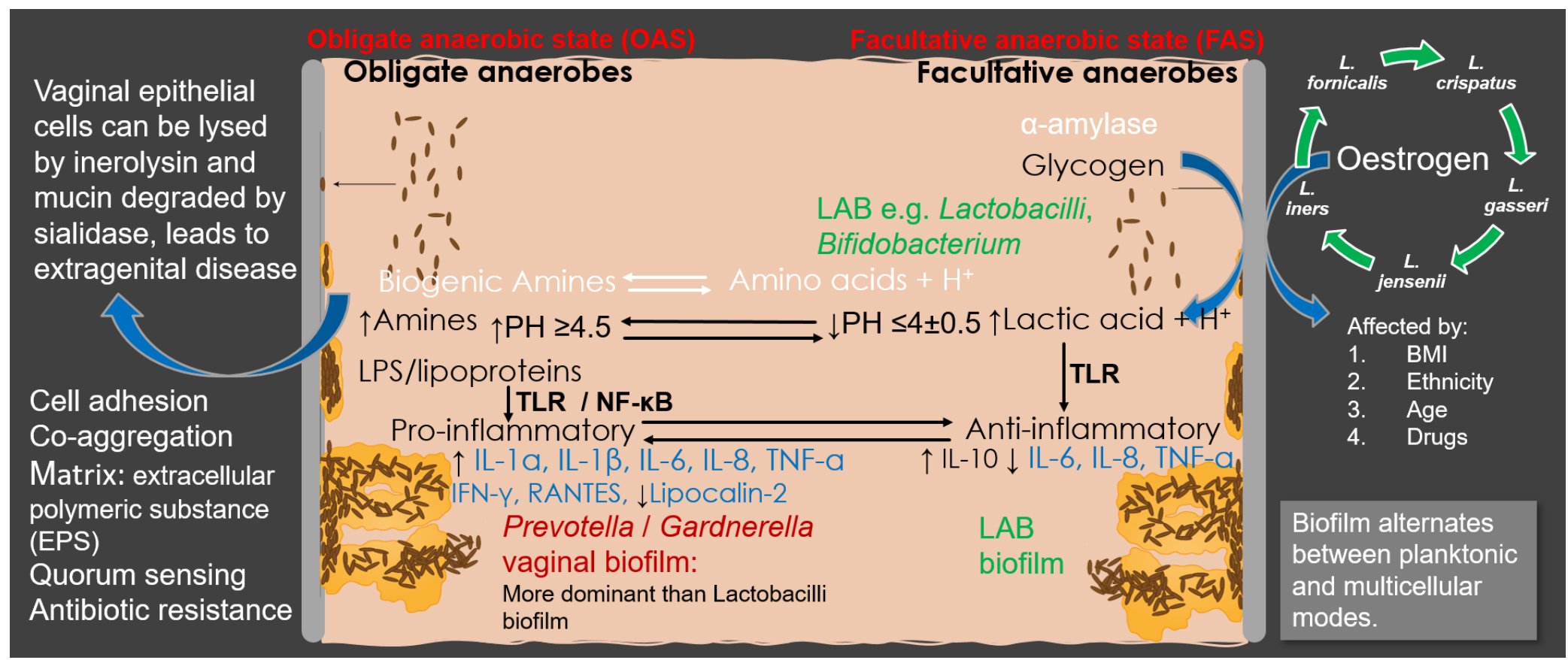

998

999

1000

1001

1002

1003

1004

1005

1006

1007

1008

1009

1010 
Taboe 1. Relative abundance for taxa in the cervicovaginal microbiome of Afro-Caribbean women based on 1012 WGS and 16S rRNA V4 sequencing.

\begin{tabular}{|c|c|c|c|}
\hline \multicolumn{2}{|l|}{ WGS sequencing (Overall $n=15$ ) } & \multicolumn{2}{|c|}{ 16S rRNA V4 sequencing (Overall $n=18$ ) } \\
\hline Taxa & Abundance (\%) & Taxa & Abundance (\%) \\
\hline Prevotella spp. & 13.91 & Prevotella & 23.06 \\
\hline Gardnerella spp. & 12.14 & Lactobacillus & 22.26 \\
\hline Lactobacillus spp. & 9.37 & Shuttleworthia & 14.00 \\
\hline Mobiluncus spp. & 7.15 & Gardnerella & 10.00 \\
\hline Sneathia spp. & 5.59 & Anaerococcus & 5.00 \\
\hline Megasphaera genomosp. type_1 str. 28L & 3.00 & Megasphaera & 4.17 \\
\hline Atopobium spp. & 2.31 & Sneathia & 4.00 \\
\hline Bifidobacterium breve DSM 20213 & 2.00 & Porphyromonas & 2.00 \\
\hline Mageeibacillus indolicus UPII9-5 & 2.00 & Dialister & 1.34 \\
\hline Porphyromonas asaccharolytica DSM 20707 & 2.00 & Atopobium & 0.86 \\
\hline Dialister micraerophilus DSM 19965 & 0.66 & Gemella & 0.52 \\
\hline Aerococcus christensenii & 0.31 & Veillonella & 0.51 \\
\hline Peptoniphilus lacrimalis DSM 7455 & 0.30 & Clostridium & 0.46 \\
\hline Eubacterium sulci ATCC 35585 & 0.23 & Peptoniphilus & 0.24 \\
\hline Propionibacterium acnes KPA171202 & 0.21 & Actinomyces & 0.21 \\
\hline Staphylococcus aureus subsp. aureus NCTC 8325 & 0.19 & Campylobacter & 0.15 \\
\hline Alloprevotella tannerae ATCC 51259 & 0.19 & Aerococcus & 0.11 \\
\hline Anaerococcus & 0.17 & Fusobacterium & 0.10 \\
\hline Streptococcus pseudoporcinus LQ 940-04 & 0.17 & & \\
\hline Thermogemmatispora carboxidivorans & 0.14 & & \\
\hline Lachnospiraceae & 0.13 & & \\
\hline Mycoplasma hominis ATCC 23114 & 0.12 & & \\
\hline Gemella asaccharolytica & 0.11 & & \\
\hline Prevotellamassilia timonensis & 0.10 & & \\
\hline
\end{tabular}

1013

1014

1015

1016 


\section{Figures}

(A)

(B)

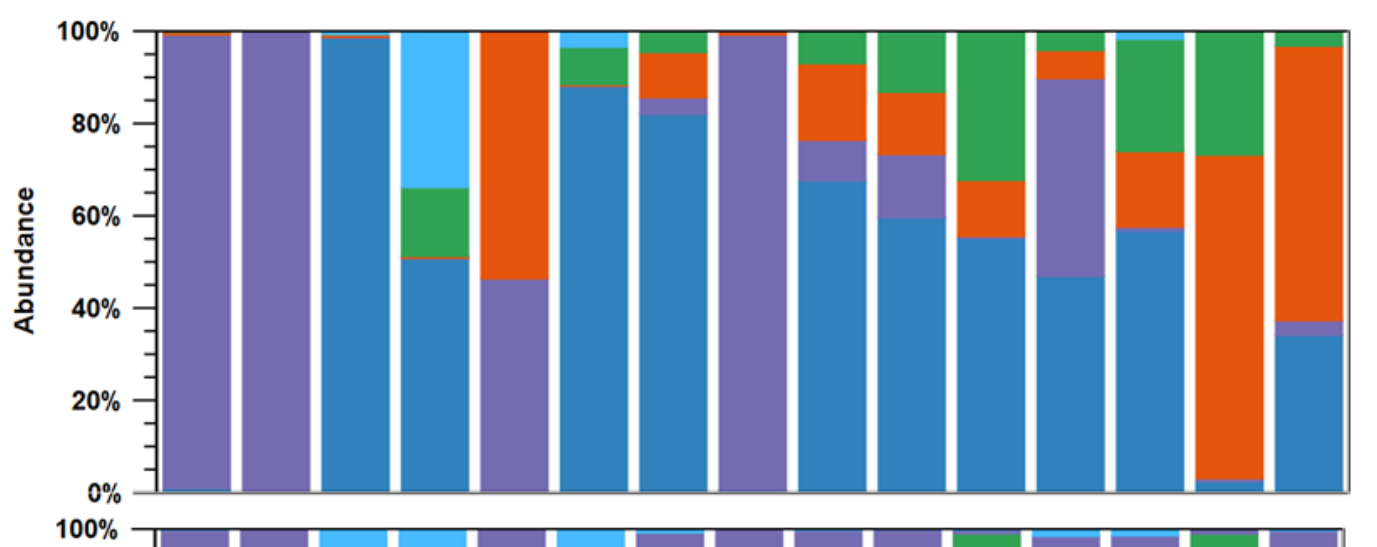

(C)

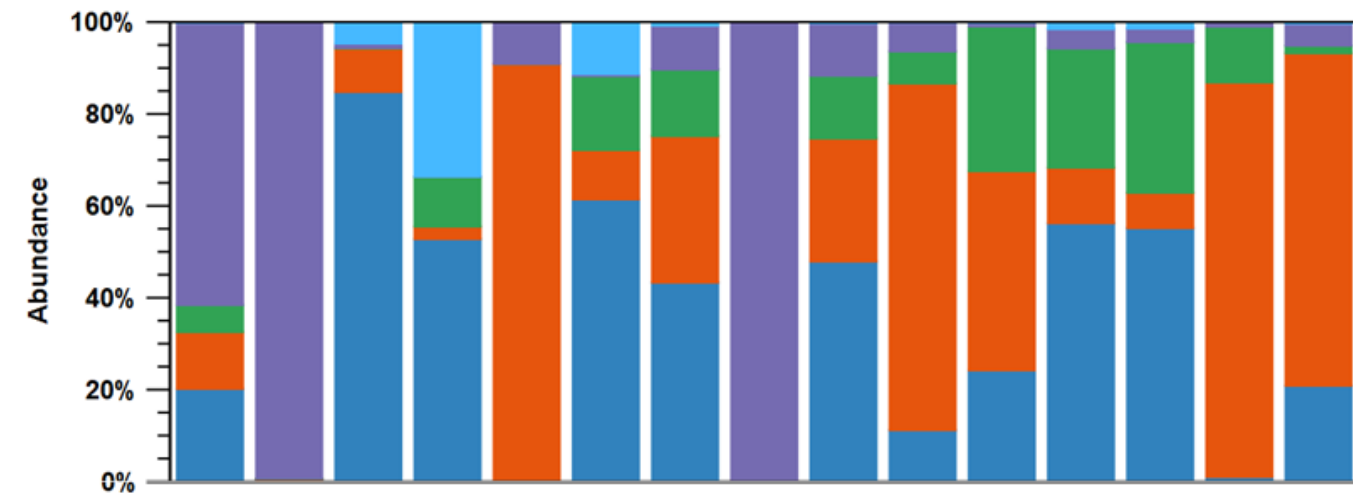

$\square$ Porphyromonas

$\square$ Lactobacillus

$\square$ Sneathia

$\square$ Gardnerella

$\square$ Prevotella
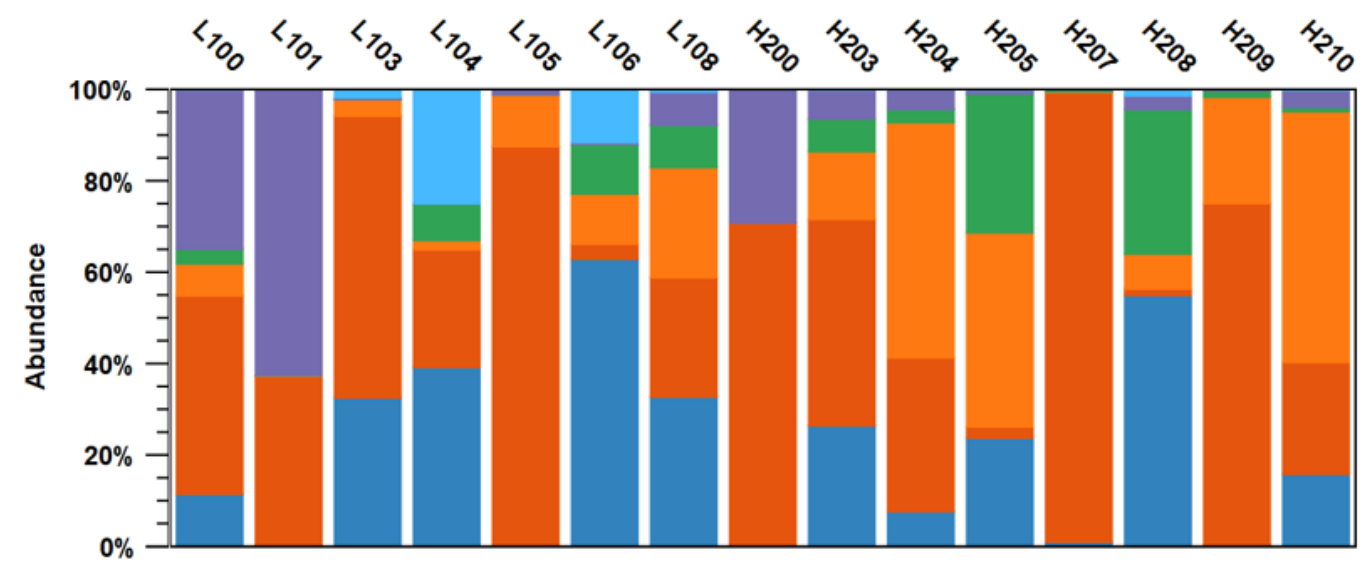

$\square$ Porphyromonas

$\square$ Lactobacillus

Sneathia

$\square$ Gardnerella

- Tetrasphaera

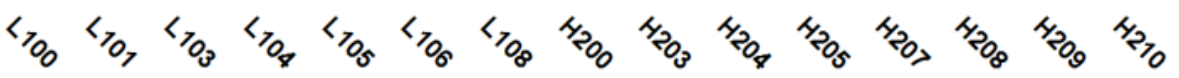

- Prevotella

\section{Figure 1}

Relative abundance of selected taxa at the species level. Microbial community structures determined by 16S rRNA V4 and WGS metagenomics are similar.(A) Relative abundance of taxa as determined by targeted 16S rRNA V4 sequencing (B) Relative abundance of taxa as determined by whole genome shotgun (relative abundances for T. japonica not included) (C) Relative abundance of taxa as determined by whole genome shotgun (relative abundances for T. japonica included). Cervicovaginal samples are labeled L100 to H210. 
(A)

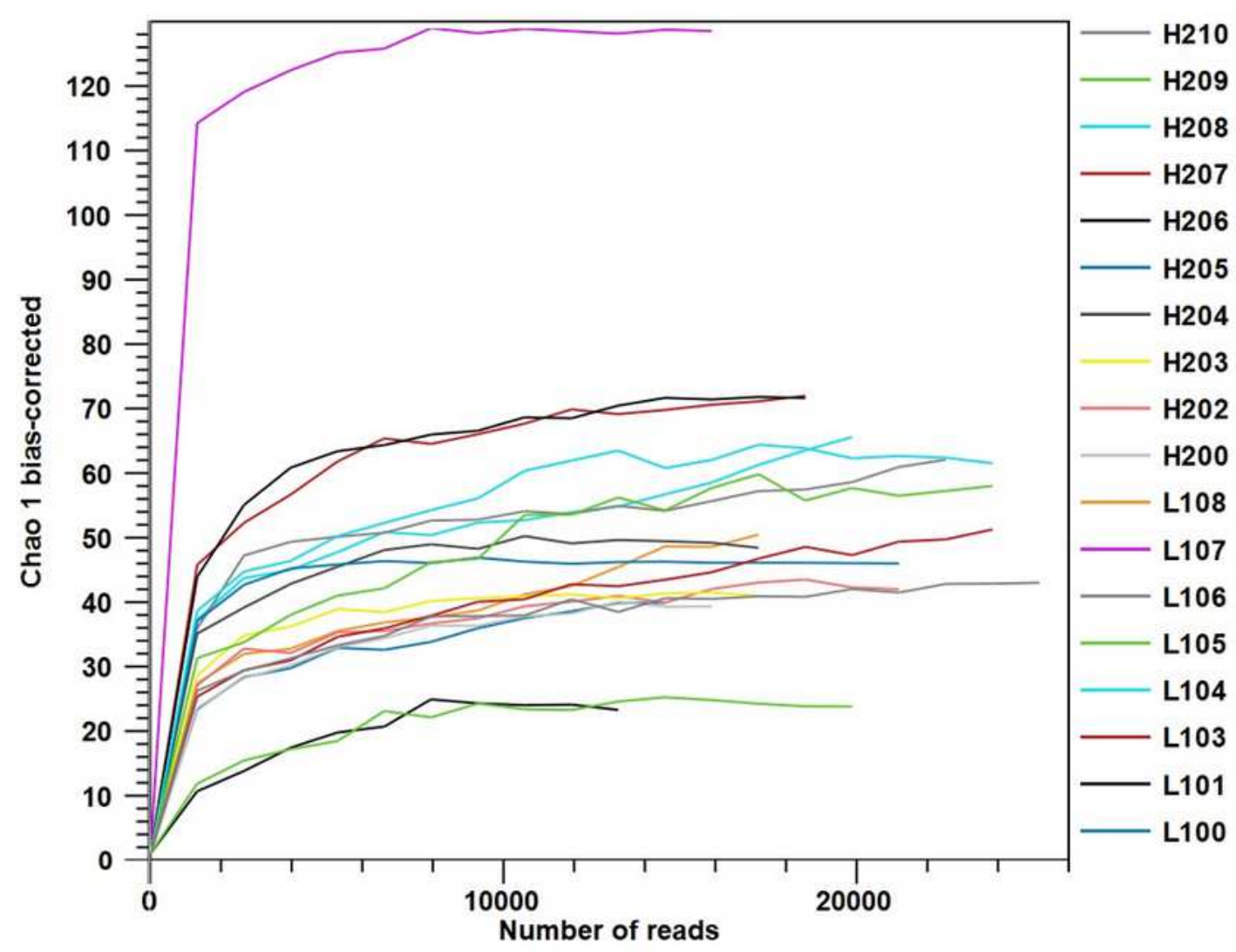

(B)

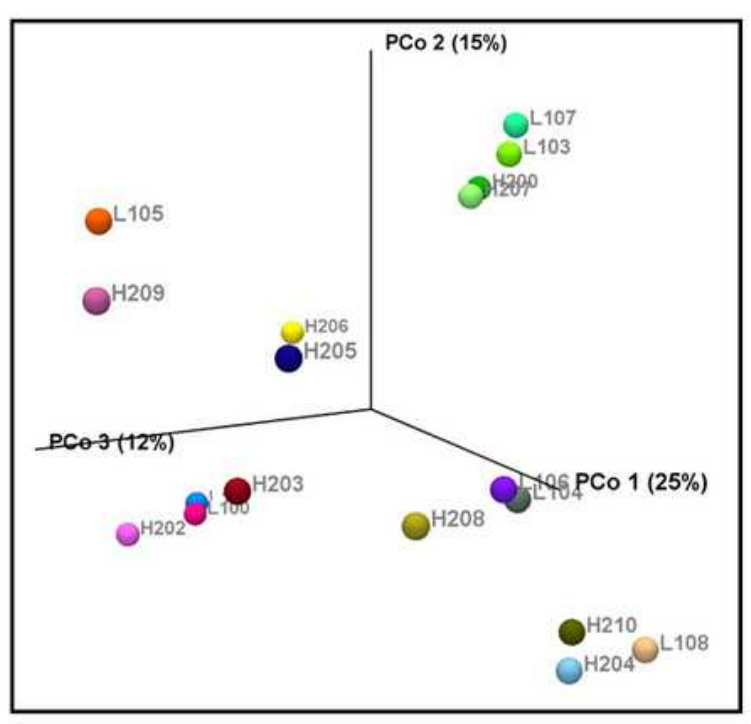

(C)

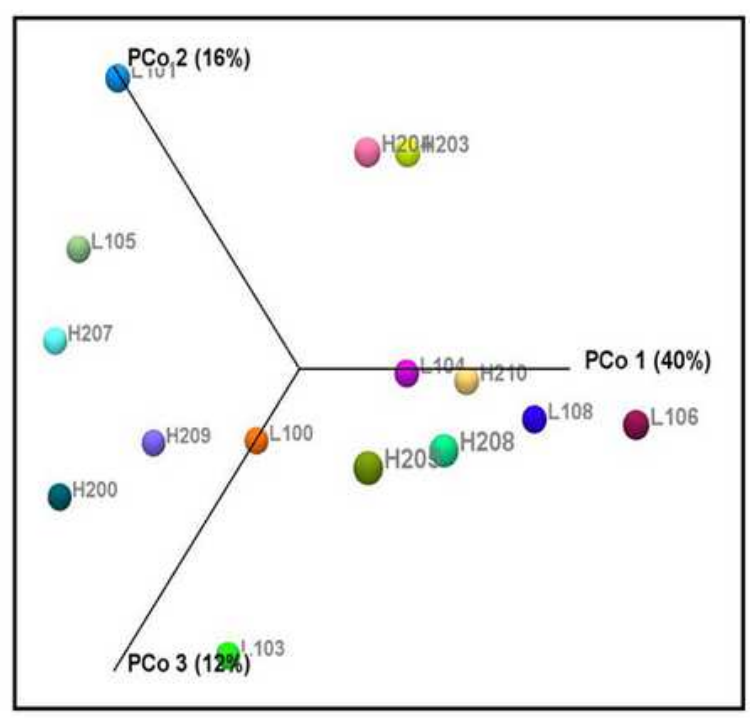

\section{Figure 2}

(A) 16S rRNA V4 sequencing data analyzed using Chao-1 bias-corrected a-diversitymetric. (B) 16S rRNA V4 sequencing data visualized with 3D principle coordinates analysis (PCoA) for using Bray-Curtis $\beta$ diversity metrics. (C) Whole genome shotgun metagenomics data visualized with 3D principal coordinates analysis (PCoA) Bray-Curtis using $\beta$-diversity metrics. Refer to supplementary Figure S1 for additional analyses. 

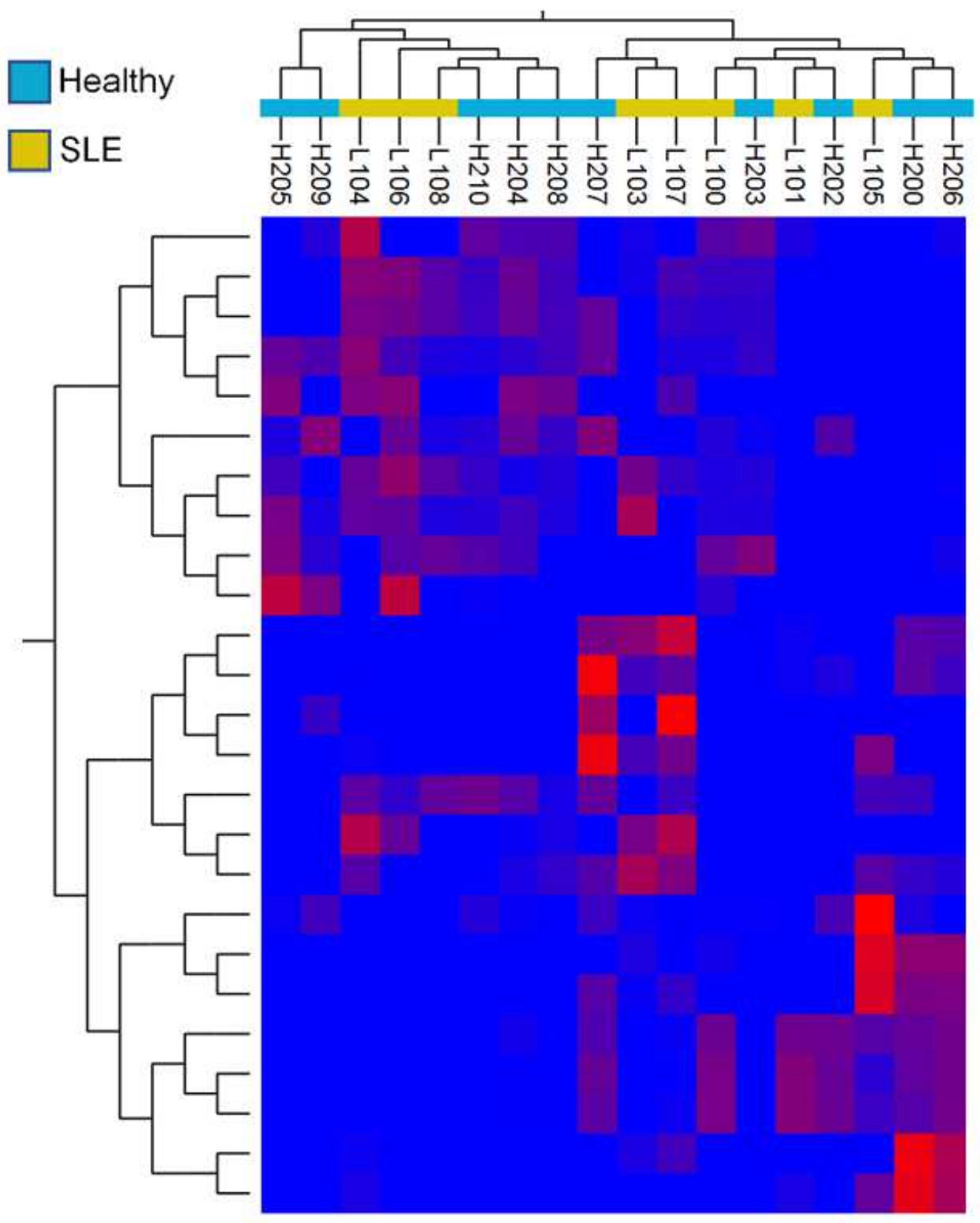

Prevotella amnii

Prevotella sp.

Prevotella sp.

Sneathia amnii

Megasphaera sp.

Sneathia sanguinegens

Prevotella sp. S7-1-8

Dialister sp.

Prevotella amnii

Prevotella timonensis

Prevotella histicola

Anaerococcus jeddahensis

Veillonella montpellierensis

Howardella $s p$.

Shuttleworthia satelles

Porphyromonas asaccharolytica

Prevotella timonensis

Gardnerella vaginalis

Lactobacillus fornicalis

Lactobacillus jensenii

Lactobacillus iners

Lactobacillus iners

Lactobacillus iners

Lactobacillus crispatus

Lactobacillus crispatus

Clustering: Sample and feature.4lustering $2.4 \quad 3.336$

Data: Normalized abundances

Dissimilarity: Euclidean distance

Linkage: Complete linkage

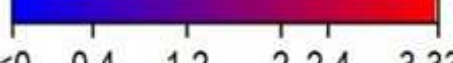

$\begin{array}{llllll}0 & 0.4 & 1.2 & 2 & 2.4 & 3.336\end{array}$

Taxon Abundance

Figure 3

Heatmap indicating relative abundance of bacteria in the cervicovaginal microbiome of Afro-Caribbean women based on 16S rRNA V4 sequence analysis. 


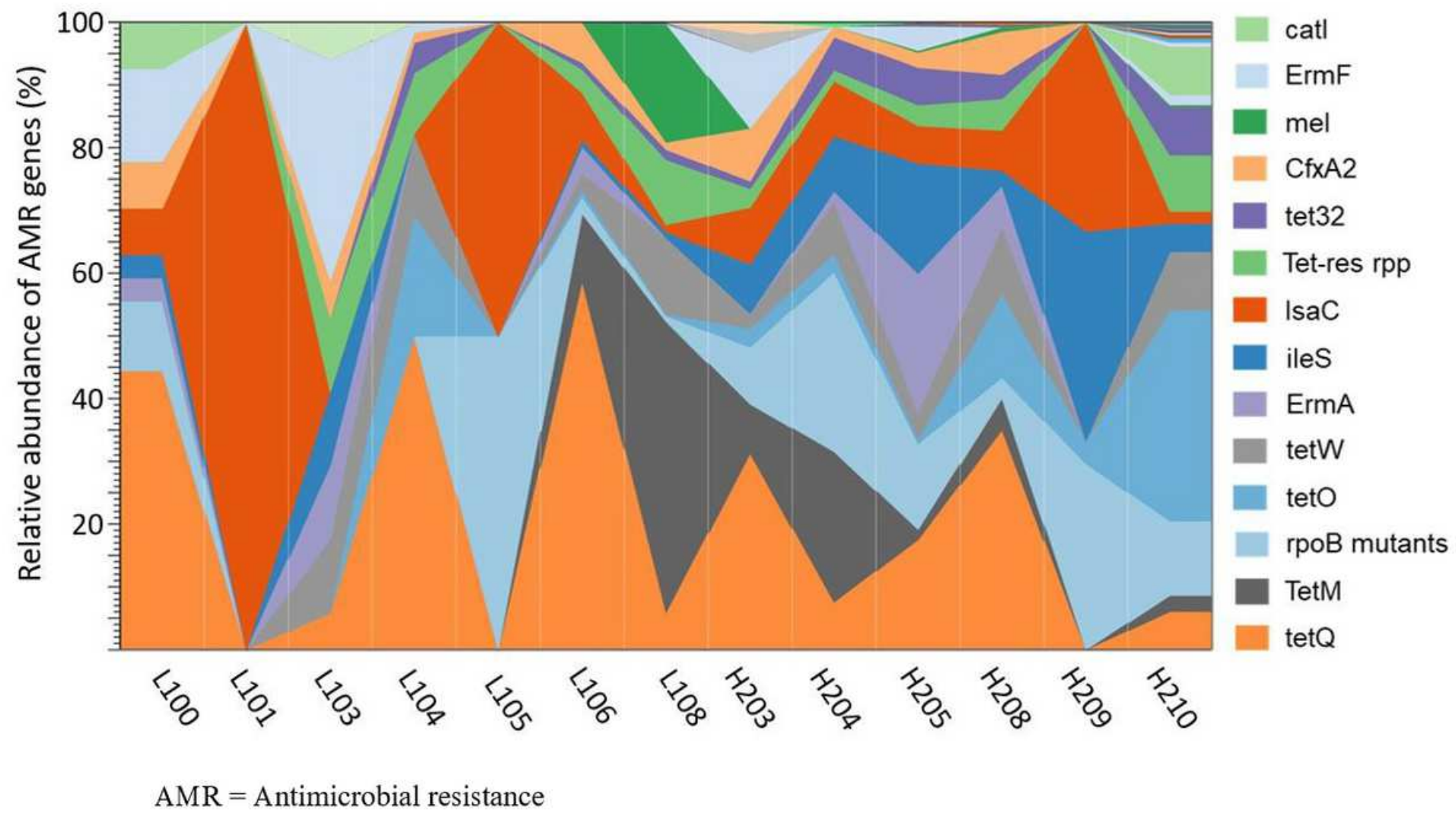

Figure 4

Area chart showing relative abundance of antimicrobial (AMR) resistance genes in the cervicovaginal microbiome of Afro-Caribbean women. 


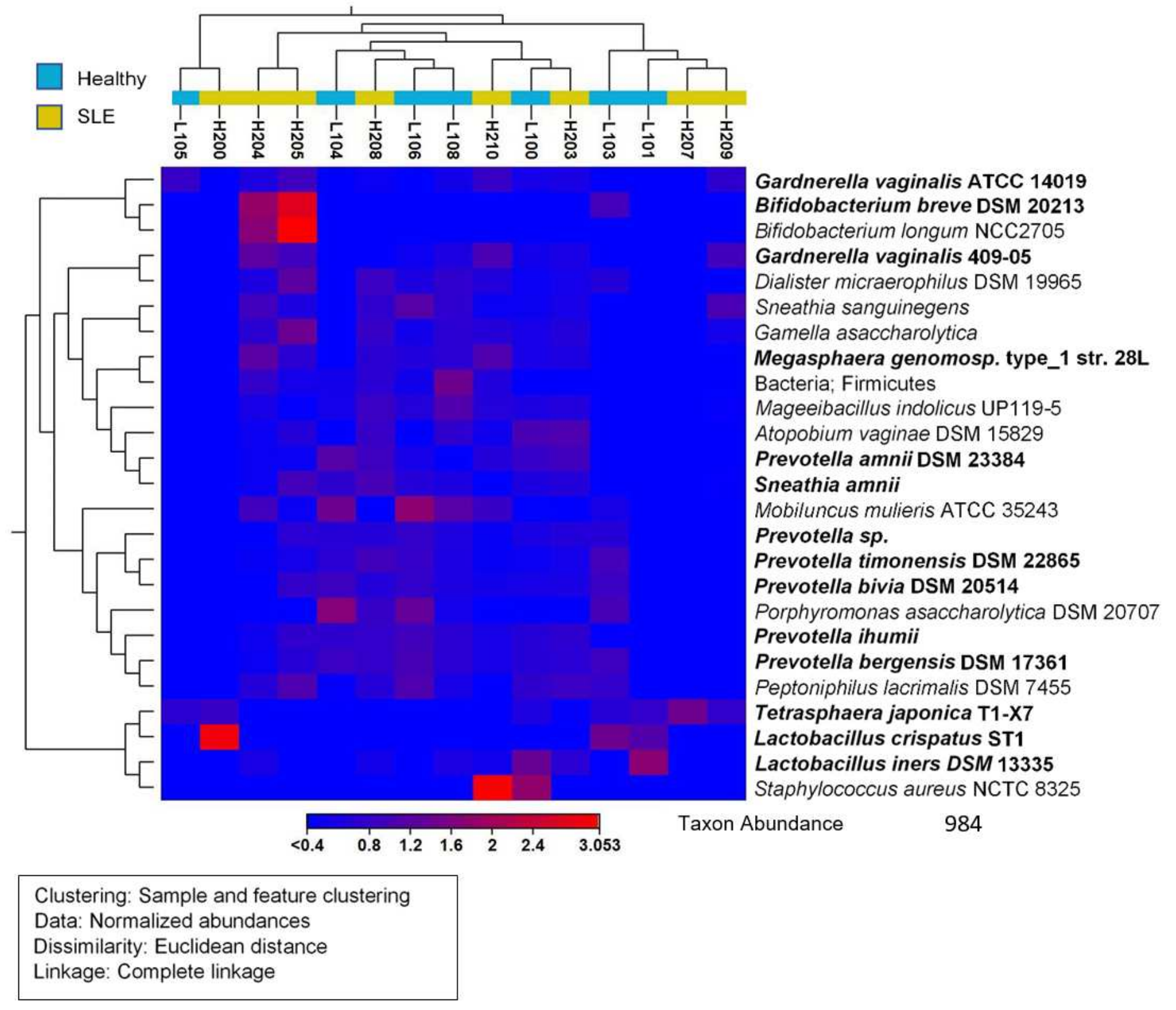

\section{Figure 5}

Heatmap indicating relative abundance of bacteria in the cervicovaginal microbiome of Afro-Caribbean women based on whole genome shotgun metagenomic sequence analysis.

\section{Figure 6}

Rooted circular cladogram of taxa of cervicovaginal microbiome of Afro-Caribbean women based on 16S rRNA V4 sequence analysis. Dominant facultative anaerobes (Lactobacillus species) and dominant obligate anaerobes (Prevotella species) are indicated by red and green boundaries, respectively. 
Vaginal epithelial cells can be lysed

by inerolysin and mucin degraded by sialidase, leads to extragenital disease

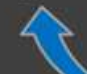

Cell adhesion

Co-aggregation

Matrix: extracellular

polymeric substance

(EPS)

Quorum sensing

Antibiotic resistance

\section{Obligate anaerobes}

1-í

i:

$-1$

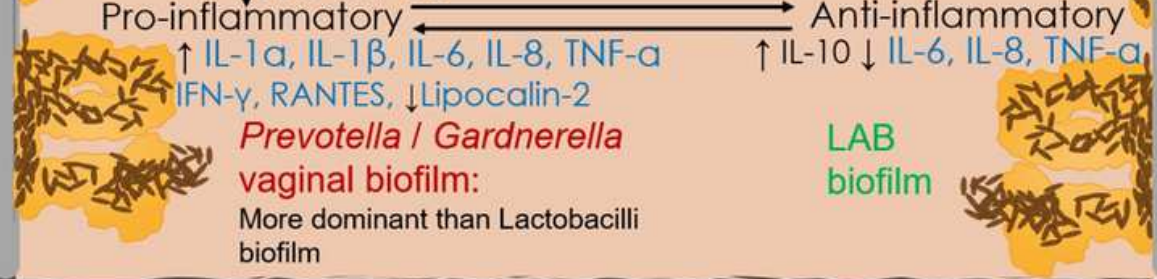

Faculfative anaerobes

LAB e.g. Lactobacilli, Bifidobacterium<smiles></smiles>

$1-1$ i,

$R$ $\downarrow \mathrm{PH} \leq 4 \pm 0.5 \uparrow$ Lactic acid $+\mathrm{H}^{+}$
1,1

(id!

$\uparrow$ Amines $\uparrow \mathrm{PH} \geq 4.5$ LPS/lipoproteins Pro-inflamm I NF-k

\section{政

tis
.

\section{Figure 7}

Schematic of the immune-munibiome model depicting the dynamic regulation of the cervicovaginal ecosystem (pink area indicates lower vaginal tract). The left border of the diagram represents the obligate anaerobe state (OAS) biofilm. On the right border is the biofilm of the facultative anaerobic state. A low $\mathrm{pH}$ (high lactic acid concentration) generated by lactic acid bacilli (LAB) bacteria is mitigated by the consumption of protons and production of biogenic amines by obligate anaerobes. Lactic acid induces an anti-inflammatory immune response, while lipopolysaccharides (LPS) and lipoproteins trigger a proinflammatory immune response. The net result is equilibrium of inflammatory responses and $\mathrm{pH}$ changes that maintains vaginal eubiosis.

\section{Supplementary Files}

This is a list of supplementary files associated with this preprint. Click to download.

- SupplementaryTableS2.OTUStatisticsTable.pdf

- SupplementaryTableS7.Metadata.xlsx

- SupplementaryTableS8.Statisticalanalysis.pdf

- SupplementaryTableS6.Mostabundanttaxa16SandWGS.xlsb

- SupplementaryTableS9.Funtionalprofile.xIsx

- SupplementaryTableS5.RelativeabundanceWGSreads.xlsx

- SupplementaryTableS4.SeqMatchresultsfor16S.pdf

- SupplementaryTableS10.Antibioticresistancegenes.xIsx

- SupplementaryTableS1.SequenceReadStatisticsTable.pdf

- SupplementaryFigureS2.Heatmapfunctionalprofiles.pdf 
- SupplementaryFigureS1.AlphaandBetadiversities.pdf

- SupplementaryTableS3.RelativeabundancevaluesandOTUs.xIsx 